\title{
Quasi-Experimental Determination of Turbulent Dispersion Parameters for Different Stability Conditions from a Tall Micrometeorological Tower
}

\author{
Luís Gustavo N. Martins, Gervásio A. Degrazia, Otávio C. Acevedo, \\ Franciano S. Puhales, Pablo E. S. De Oliveira, Claudio A. Teichrieb, and \\ SAMUEL M. DA SILVA \\ Deptamento de Física, Universidade Federal de Santa Maria, Santa Maria, Brazil
}

(Manuscript received 26 September 2017, in final form 19 April 2018)

\begin{abstract}
Turbulent wind data measured by sonic anemometers installed at various heights on a 140 -m-tall micrometeorological tower located at a coastal site are used to obtain vertical profiles of the velocity standard deviations $\sigma_{i}$, Lagrangian decorrelation local time scales $T_{L i}$, and eddy diffusivities $K_{\alpha}$ for distinct stability conditions. The novelty of the study lies in the use of turbulent data directly measured over the extension of the atmospheric surface layer at a coastal site for that purpose. Furthermore, the approach employs the Hilbert-Huang transform to determine the wind energy spectral peak frequencies. These are applied to the asymptotic spectral equation from Taylor statistical diffusion theory to obtain the turbulent dispersion parameters, which are shown to generally agree well with those provided by a classical autocorrelation approach. For neutral and stable situations the vertical profiles of momentum eddy diffusivities agree well with those derived from the spectral and autocorrelation method. Additionally, the turbulent integral time scales and eddy diffusivities determined by the method at a coastal location are found to overestimate those predicted from analytical expressions based on continental field observations. The turbulence parameters found are suitable to be employed in air pollution dispersion models.
\end{abstract}

\section{Introduction}

Turbulent dispersion parameters play a fundamental role in air pollution dispersion models. In such models, the turbulent concentration fluxes (in Eulerian models) and the stochastic term associated with pressure fluctuations (in Lagrangian models) must be parameterized to properly represent the real atmospheric dispersion. Both historically and presently, the most important challenges in the field of atmospheric dispersion lie on properly parameterizing such turbulent terms in a physically consistent manner. Generally, to quantify the turbulent diffusion in Eulerian models, a first-order scheme employs eddy diffusivities $\left(K_{\alpha}, \alpha=x, y, z\right)$ that relate the turbulent fluxes to the mean gradients of the quantities being transferred (Garratt et al. 1992). They have dimensions of length times velocity, which therefore represent the eddy size and eddy velocity (Panofsky and Dutton 1983). Lagrangian models, on the other hand, use $T_{L i}$, which is a measure of the longest

Corresponding author: Luís Gustavo N. Martins, lgnm.sm@ gmail.com time during which, on average, a fluid particle persists in motion in a given direction (Hinze 1975) and the turbulent velocity variances $\left(\sigma_{i}^{2}, i=u, v, w\right)$, which represent a quantification of the turbulent mixing, therefore identifying the flow ability to disperse contaminants. For inhomogeneous turbulence, $T_{L i}$ is termed a local velocity decorrelation time scale (Rodean 1996). Therefore, Eulerian and Lagrangian dispersion models, which are based, respectively, upon the well-known advectiondiffusion and Langevin equations, need to describe the eddy motions associated with the unknown terms. These terms are responsible by the turbulent transport and they are typically represented in terms of eddy diffusivities, Lagrangian local decorrelation time scales, and turbulent velocity variances (Gryning et al. 1983; Sawford 1985; Thomson 1987; Rodean 1996; Degrazia et al. 2000a; Ulke 2000; Sharan and Modani 2006; Degrazia et al. 2007; Leelössy et al. 2014). Comprehensive reviews on the subject of air pollution dispersion have been provided by Steeneveld and Holtslag (2009) and by Leelössy et al. (2014).

A 140-m micrometeorological tower, fully instrumented with 11 levels of observations of the turbulent 
wind components and temperature, has been operating in southeastern Brazil since August 2016, at a coastal site, located $4 \mathrm{~km}$ from the coastline (Acevedo et al. 2018). In the present study, these measurements are used to determine turbulent dispersion parameters such as $K_{\alpha}$ and $T_{L i}$, with the use of a few mathematical expressions. The main novelty of the approach lies in the fact that the dispersion parameters are locally determined from the observations made across the entire extension of the atmospheric surface layer. For this reason, the method is referred as quasi-experimental. It contrasts with previous studies in which the vertical profiles are related to surface observations through similarity-based relationships (Hanna 1982; Holtslag and Moeng 1991; Ulke 2000; Degrazia et al. 2001; Wyngaard 2010).

From the tower observations, two different approaches are used to infer the turbulent dispersion parameters. The first one is spectrally based, while the other is based on the estimation of the autocorrelation function. Additional goals of the study are to provide a direct comparison between these two approaches, as well as comparing them to equivalent quantities inferred from expressions from the literature, which depend on typical length and velocity scales. The methodology employed for the spectral analysis is based on the Hilbert-Huang transform and is used to identify the frequencies associated with the spectral peaks. In a statistical sense, these particular frequencies determine the physical characteristics (time and length scales) associated with the energy-containing eddies.

\section{Turbulent parameterization applied to air pollution dispersion models}

Eulerian air pollution dispersion models are based in the mass continuity equation and employ the so-called advection-diffusion equation (Hanna 1982; Blackadar 1997) to simulate the observed contaminant concentrations. Generally, to parameterize the unknown contaminant turbulent fluxes that arise in the advection-diffusion equation, the turbulent parameterization models are based on the gradient transport hypothesis and employ $K_{\alpha}$ to describe the turbulent diffusive character acting on pollutants released in the PBL. On the other hand, Lagrangian stochastic particle models that also describe the air pollution concentrations are based on the Langevin equation. The generalized form of these Lagrangian dispersion models is composed by a deterministic term describing the viscosity effect of the turbulent flow acting on the fluid particles and a stochastic term associated with the pressure fluctuations (provoked by the turbulent velocity variations) acting on these particles. These unknown terms, forming the Lagrangian dispersion models, are parameterized by mathematical relations described in terms of $\sigma_{i}^{2}$ and $T_{L i}$ (Rodean 1996; Anfossi and Physick 2005).

Simple expressions for eddy diffusivities and the local decorrelation time scales

Employing Taylor's statistical diffusion theory, Batchelor (1949) and Pasquil and Smith (1983) derived the expressions for the eddy diffusivities for an ensemble of particles for any travel time $t$ :

$$
K_{\alpha}=\frac{\sigma_{i}^{2} \beta_{i}}{2 \pi} \int_{0}^{\infty} F_{i}^{E}(n) \frac{\sin \left(2 \pi n t / \beta_{i}\right)}{n} d n
$$

where

$$
\beta_{i}=\frac{T_{L i}}{T_{E i}}=0.55 \frac{U}{\sigma_{i}}
$$

is defined as the ratio of the Lagrangian to the Eulerian decorrelation (or integral) time scales (Gifford 1955; Wandel and Kofoed-Hansen 1962; Degrazia and Anfossi $1998), U$ is the horizontal mean wind speed, and $F_{i}^{E}(n)$ is the Eulerian spectrum of energy $\left[S_{i}^{E}(n)\right]$ normalized by $\sigma_{i}^{2}$; $n$ is the frequency.

For large diffusion travel times $\left(t \gg T_{L i}\right)$, the following analysis can be developed. Considering that $F_{i}(n)$ and $\sin \left(2 \pi n t / \beta_{i}\right) / n$ are even functions of $n$, Eq. (1) can be written as

$$
K_{\alpha}=\frac{\sigma_{i}^{2} \beta_{i}}{4} \int_{-\infty}^{\infty} \frac{F_{i}^{E}(n) \sin \left(2 \pi n t / \beta_{i}\right)}{\pi n} d n .
$$

Defining $g=2 \pi n / \beta_{i}$, the above equation can be rewritten in the form

$$
K_{\alpha}=\frac{\sigma_{i}^{2} \beta_{i}}{4} \int_{-\infty}^{\infty} \frac{F_{i}^{E}\left(\frac{g \beta_{i}}{2 \pi}\right) \sin (g t)}{\pi g} d g .
$$

For $t \rightarrow \infty$, Eq. (4) yields

$$
K_{\alpha}=\frac{\sigma_{i}^{2} \beta_{i}}{4} \int_{-\infty}^{\infty} F_{i}^{E}\left(\frac{g \beta_{i}}{2 \pi}\right) \lim _{t \rightarrow \infty} \frac{\sin (g t)}{\pi g} d g,
$$

where $\lim _{t \rightarrow \infty} \sin (g t) / \pi g$ is a well-known representation of a Dirac delta function. Then,

$$
\begin{aligned}
K_{\alpha} & =\frac{\sigma_{i}^{2} \beta_{i}}{4} \int_{-\infty}^{\infty} F_{i}^{E}\left(\frac{g \beta_{i}}{2 \pi}\right) \delta(g) d g \\
& =\frac{\sigma_{i}^{2} \beta_{i}}{4} F_{i}^{E}(n \rightarrow 0)=\sigma_{i}^{2} T_{L i},
\end{aligned}
$$

where $\delta g$ is the Dirac delta function and 


$$
T_{L i}=\frac{\beta_{i}}{4} F_{i}^{E}(n \rightarrow 0) .
$$

Equations (6) and (7) are described in terms of the energy spectra at the origin.

The following expressions derived by Degrazia et al. (2000a,b) represent models used to describe measured turbulent spectra in a convective and shear-driven (stable and neutral conditions) PBL:

$$
\frac{n\left(S_{i}^{E}\right)^{c}}{w_{*}^{2}}=\frac{1.06 c_{i} f\left(\psi_{\varepsilon} \frac{z}{z_{i}}\right)^{2 / 3} f}{\left[1+\frac{1.5 f}{\left(f_{m}\right)_{i}^{c}}\right]^{5 / 3}\left[\left(f_{m}\right)_{i}^{c}\right]^{5 / 3}}
$$

and

$$
\frac{n\left(S_{i}^{E}\right)^{n, s}}{u_{*}^{2}}=\frac{1.5 c_{i} f \phi_{\varepsilon}^{2 / 3}}{\left\{1+\frac{1.5 f^{5 / 3}}{\left[\left(f_{m}\right)_{i}^{n, s}\right]^{5 / 3}}\right\}\left[\left(f_{m}\right)_{i}^{n, s}\right]^{5 / 3}},
$$

where $w_{*}$ is the convective velocity scale, $f=n z / U$ is the normalized frequency, $z$ is the height above the surface, $(\mathrm{fm})_{i}^{c}$ is the normalized frequency of the convective spectral peak, $\psi_{\varepsilon}=\varepsilon_{b} z_{i} / w_{*}^{3}$ is the adimensional dissipation rate function, $\varepsilon_{b}$ is the buoyant rate of turbulent kinetic energy (TKE) dissipation, $z_{i}$ is the convective boundary layer (CBL) height, $u_{*}$ is the friction velocity, $(f m)_{i}^{n, s}$ is the normalized frequency of the neutral or stable spectral peak, the dissipation rate $\phi_{\varepsilon}=\varepsilon_{s} k z / u_{*}^{3}$ is adimensionalized with surface layer scaling parameters, $\varepsilon_{s}$ is the mechanical rate of TKE dissipation, $\kappa$ is the von Kármán constant, and $c_{i}=\gamma_{i} \gamma_{u}(2 \pi)^{-2 / 3}$, with $\gamma_{u}=0.5 \pm 0.05$, and $\gamma_{i}=1,4 / 3$, and $4 / 3$ for $u, v$, and $w$ components, respectively (Champagne et al. 1977; Sorbjan 1989).

Analytical integration of Eqs. (8) and (9) over the whole frequency domain leads to the following turbulent velocity variances:

$$
\left(\sigma_{i}^{c}\right)^{2}=\frac{1.06 c_{i} \psi_{\varepsilon}^{2 / 3} w_{*}^{2}\left(\frac{z}{z_{i}}\right)^{2 / 3}}{\left[\left(f_{m}\right)_{i}^{c}\right]^{2 / 3}}
$$

and

$$
\left(\sigma_{i}^{n, s}\right)^{2}=\frac{2.32 c_{i} \phi_{\varepsilon}^{2 / 3} u_{*}^{2}}{\left[\left(f_{m}\right)_{i}^{n, s}\right]^{2 / 3}}
$$

which are employed to normalize the spectra [Eqs. (8) and (9)]. Thus, the normalized spectra can now be written as

$$
\left(F_{i}^{E}\right)^{c}(n \rightarrow 0)=\frac{\left(S_{i}^{E}\right)^{c}(n \rightarrow 0)}{\left(\sigma_{i}^{c}\right)^{2}}=\frac{z}{\left(f_{m}\right)_{i}^{c} U}
$$

and

$$
\left(F_{i}^{E}\right)^{n, s}(n \rightarrow 0)=\frac{\left(S_{i}^{E}\right)^{n, s}(n \rightarrow 0)}{\left(\sigma_{i}^{n, s}\right)^{2}}=\frac{0.64 z}{\left(f_{m}\right)_{i}^{n, s} U} .
$$

Employing Eqs. (12) and (13), together with $\beta_{i}$ in Eqs. (6) and (7) yields, for different stability conditions, the following expressions for the eddy diffusivities and turbulent local decorrelation time scales:

$K_{\alpha}^{c}=\frac{0.55 \sigma_{i} z}{4\left(f_{m}\right)_{i}^{c}} \quad$ (convective or unstable conditions),

$K_{\alpha}^{n, s}=\frac{0.35 \sigma_{i} z}{4\left(f_{m}\right)_{i}^{n, s}} \quad$ (neutral or stable conditions),

$T_{L i}^{c}=\frac{0.55 z}{4 \sigma_{i}\left(f_{m}\right)_{i}^{c}} \quad$ (convective or unstable conditions),

and

$T_{L i}^{n, s}=\frac{0.35 z}{4 \sigma_{i}\left(f_{m}\right)_{i}^{n, s}} \quad$ (neutral or stable conditions).

Alternatively, the time scales $T_{L i}$ can be obtained using $\beta_{i}$ [Eq. (2)], where $T_{E i}$ is calculated directly from the following classical expression (Hanna 1981):

$$
T_{E i}=\int_{0}^{\infty} \frac{1}{\sigma_{i}^{2}}\left[\overline{u_{i}^{\prime}(t) u_{i}^{\prime}(t+\tau)}\right] d \tau,
$$

where $\tau$ is an incremental time lag. Furthermore, the experimental methodology provided by Eqs. (18) and (2) allows us to obtain the eddy diffusivities from the relationship $K_{\alpha}=\sigma_{i}^{2} T_{L i}$. Therefore, Eqs. (18) and (2) allow us to validate the new method to calculate the turbulent parameters expressed in Eqs. (14)-(17).

To obtain the magnitudes of $K_{\alpha}$ and $T_{L i}$, one needs the measured values of the velocity standard deviations and the spectral peak frequencies. In the present study, the spectral peak frequencies are estimated from the Hilbert-Huang spectra obtained directly from the measured wind velocity data. Furthermore, $\sigma_{i}^{2}$ and $T_{E i}$ are also calculated from the turbulent velocity data measured by the sonic anemometers.

In previous studies, the spectral peak frequencies were often found from similarity expressions obtained from a few classical studies that performed observations over the entire depth of the PBL (Kaimal et al. 1972, 1976; Caughey and Palmer 1979). 


\section{Micrometeorological observations and methodology}

Turbulent measurements were performed at a $140-\mathrm{m}$ micrometeorological tower located in the city of Linhares, Brazil $\left(19^{\circ} 32^{\prime} 01^{\prime \prime} \mathrm{S}, 39^{\circ} 48^{\prime} 04^{\prime \prime} \mathrm{W}\right)$. High-frequency $(10 \mathrm{~Hz})$ measurements of the components of the wind velocity and temperature are carried out at heights of 2, 5, 9, 20, $37,56,75,94,113$, and $132 \mathrm{~m}$ by 10 tridimensional sonic anemometers; at the 20 -m level by an IRGASON sonic anemometer (Campbell Scientific, Inc.); and at the other levels by a CSAT3B sonic anemometer (Campbell Scientific). The tower is located $240 \mathrm{~m}$ north of a natural gas power plant and $4 \mathrm{~km}$ southwest of the ocean (coastal region). The inland terrain is flat within $30 \mathrm{~km}$ from the tower, an area that is covered with sparse shrub vegetation. Details on the local characteristics, as well as maps and pictures of the site, can be found in Acevedo et al. (2018). Only periods without errors in the anemometers diagnostic control flags at all levels have been retained. Periods with southerly winds, which blow from the power plant to the tower, are rejected. Each 1-h segment has been rotated to the preferential wind direction using the double-rotation method (Kaimal and Finnigan 1994).

The Obukhov length $(L)$, friction velocity $\left(u_{*}\right)$, and mean wind speed $(U)$ are used to group the intervals in three stability conditions of the PBL. The $L$ parameter, described as the ratio between the mechanical and convective forcings, can be written as

$$
L=\frac{-\bar{\theta} u_{*}^{3}}{\kappa g \overline{w^{\prime} \theta^{\prime}}},
$$

where $u_{*}=\left[\left(\overline{u^{\prime} w^{\prime}}\right)^{2}+\left(\overline{v^{\prime} w^{\prime}}\right)^{2}\right]^{1 / 4}, g$ is the gravity acceleration, $\bar{\theta}$ is the mean potential temperature, and $\overline{w^{\prime} \theta^{\prime}}$ is the turbulent kinematic heat flux. These parameters have been used to classify the stability conditions into three classes, based on observations made at $10 \mathrm{~m}$ (Table 1). Under stable conditions, recent studies have shown a sharp contrast between very stable and weakly stable conditions (Mahrt 1998; Sun et al. 2012). The very stable case is not addressed here, as turbulence is not sustainable in this situation (Van de Wiel et al. 2012), and the stable boundary layer may be very shallow. The transition between the two regimes typically happens at $0.05<z / L<0.2$ (Mahrt 1998; Acevedo et al. 2014), so that $L=100 \mathrm{~m}$ has been chosen as the upper limit for this variable. Mortarini et al. (2016) have shown that weak wind conditions, when the flow is severely affected by nonturbulent fluctuations such as meandering, usually happen when the mean wind speed $(U)$ is less than $1.5 \mathrm{~m} \mathrm{~s}^{-1}$. Therefore, a lower limit of $2.0 \mathrm{~m} \mathrm{~s}^{-1}$ has been
TABLE 1. Parameters used to characterize the stability conditions of the ABL.

\begin{tabular}{lcccc}
\hline \hline Conditions & $L(\mathrm{~m})$ & $u_{*}\left(\mathrm{~m} \mathrm{~s}^{-1}\right)$ & $U\left(\mathrm{~m} \mathrm{~s}^{-1}\right)$ & $\begin{array}{c}\text { No. of } \\
\text { intervals }\end{array}$ \\
\hline Unstable & $-150 \leq L<0$ & - & - & 343 \\
Neutral & $\geq 300$ & $\geq 0.4$ & $\geq 4$ & 123 \\
Stable & $0<L \leq 100$ & $\leq 0.3$ & $2 \leq U<4$ & 22 \\
\hline
\end{tabular}

imposed for $U$ at stable conditions. Neutral conditions have been assumed when $U>4.0 \mathrm{~m} \mathrm{~s}^{-1}, u_{*}>0.4 \mathrm{~m} \mathrm{~s}^{-1}$ (Garratt et al. 1992), and $L>300 \mathrm{~m}$, to avoid mixed or transitional situations. For this reason, neutral conditions with negative $L$ values are not considered, as $72 \%$ of the cases with $L<-300 \mathrm{~m}$ happen in the late afternoon transitional period.

\section{Hilbert-Huang transform}

In this study we utilize the Hilbert-Huang transform to evaluate the turbulent spectra and, therefore, to identify the peak frequencies associated to the energycontaining eddies. Such frequencies are employed in Eqs. (14)-(17) to calculate $K_{\alpha}$ and $T_{L i}$.

The Hilbert-Huang transform is a time-frequency method of analysis based on a well-known method of sparse representation called empirical mode decomposition (EMD) and the Hilbert transform (Huang et al. 1998). Sparse representation is a family of methods from which an original signal is represented as a sum of a limited and small number of appropriate simple functions (Young et al. 2017). The EMD is a data-driven decomposition based on the local properties of the signal and, for this reason, can be applied to nonlinear and nonstationary signals, such as the turbulent measurements. The EMD is applied to a signal to decompose it in a set of functions, called intrinsic mode functions (IMFs). The Hilbert transform of these functions provides instantaneous frequency with real physical meaning.

EMD consists of a simple and iterative process: (i) identify the local maxima and minima points of a measured signal $x(t)$, (ii) build an upper-lower border of an envelope that covers the signal linking the maximaminima points, (iii) take the local average $(m)$ of the envelope, and (iv) the first IMF candidate $\left(h_{1}\right)$ is obtained by removing $m$ from the measured signal $[x(t)]$. The procedure is applied repeatedly to each candidate $h_{k}$ until a predetermined stoppage criteria is fulfilled (Huang et al. 1999; Rilling et al. 2003). At this point, $h_{k}$ is assigned as the first IMF $\left(C_{1}\right)$ that represents the higherfrequency natural mode of oscillation contained in $x(t)$. The first residue $\left(r_{1}\right)$ is evaluated by removing $C_{1}$ from $x(t)$. The other IMFs (lower frequencies) are obtained 
by applying steps i-iv above sequentially in each newer residue $\left(r_{k}\right)$ until no IMFs can be extracted from the residual. This last residual component $\left(r_{n}\right)$ is assigned as the residue of the EMD and represents the natural trend of the signal. With this, $x(t)$ can be expressed as

$$
x(t)=\sum_{i=1}^{N} C_{i}(t)+r_{n}(t)
$$

The Hilbert transform is applied to each $\operatorname{IMF}\left(C_{i}\right)$ as follows:

$$
\hat{C}(t)=\frac{1}{\pi} P \int_{-\infty}^{\infty} \frac{C\left(t^{\prime}\right)}{t-t^{\prime}} d t^{\prime},
$$

where $P$ indicates the Cauchy principal value.

With the pair of transforms $(C$ and $\hat{C})$, an analytic signal can be defined

$$
Z(t)=C(t)+i \hat{C}(t)=a(t) e^{i \theta(t)},
$$

where

$$
a(t)=\left[C^{2}(t)+\hat{C}_{i}^{2}(t)\right]^{1 / 2} \quad \text { and } \quad \theta(t)=\arctan \left[\frac{\hat{C}_{i}(t)}{C(t)}\right]
$$

represent, respectively, the amplitude and phase of the analytic signal.

Last, the instantaneous frequency can be evaluated as

$$
\omega=\frac{d \theta(t)}{d t} .
$$

The time-frequency distribution of the amplitude defines the Hilbert-Huang spectra $[H(\omega, t)]$. The integral over the whole period $T$ of the Hilbert-Huang spectra is the so-called Hilbert-Huang marginal spectral $S(\omega)$; so,

$$
S(\omega)=\int_{0}^{T} H(\omega, t) d t .
$$

The spectral analysis derived from Taylor statistical diffusion theory is formulated based on the classical Fourier spectral representation. According to Eq. (25), the Hilbert-Huang marginal spectra are also a representation of the turbulent energy in the frequency space. So, there is no restriction for the substitution of the Fourier transform by the Hilbert-Huang representation to identify the spectral peak $\left(f_{m}\right)$ associated with energy containing eddies. For this task, there are advantages to using the marginal Hilbert-Huang spectra. As discussed by Martins et al. (2016), the Fourier spectrum presents a rough resolution of the lowest frequencies compared with the region of the highest frequencies. Consequently, the frequency of occurrence of spectral peaks in the low-frequency region when evaluated from the Fourier transform is compromised, since the transform provides only a few sparse values. In the Hilbert-Huang spectra, on the other hand, the frequency resolution depends on the number of bins in which the set of amplitudes is operationally represented. This can be done in such a way that the resolution is constant in all of the frequency space. Furthermore, the Hilbert transform is applied only in the set of decomposed IMFs, excluding the residue $\left(r_{n}\right)$. For this reason, the Hilbert-Huang spectra are a representation of a detrended signal. Huang et al. (1998) presented a detailed comparison between Hilbert-Huang and other types of signal decomposition, such as wavelet spectra, discussing the advantages and disadvantages of the techniques.

To reduce the edge effect that mainly affects the determination of the low-frequency modes in the HilbertHuang spectra evaluated for 1 -h intervals, the EMD is applied to 3-h joined intervals. The composed interval is constituted by the main series and its previous and posterior 1-h set of measurements. The Hilbert transform is applied only to the central 1-h interval. The Hilbert-Huang marginal spectra are represented in a set of $128 \mathrm{log}$-spaced bins of frequency. This fact, therefore, imposed an additional restriction that three successive hours of good data at all levels exist.

From a total of four months of observations (August-November 2016), 795 intervals satisfy all of the requirements.

\section{Results}

Atmospheric boundary layer flow is composed of a wide range of eddy motions, with distinct scales, and is constantly subject to several external factors, such as the presence of both turbulent and nonturbulent events. For this reason, atmospheric turbulent energy spectra do not always present a well-behaved shape, so that it may be difficult to identify a clear spectral peak. To avoid this shortcoming, it is usual to average a set of characteristic spectra to obtain a smooth curve, which represents the averaged distribution of energy. This procedure is followed in the present study. Figures 1-3 show, respectively, the vertical variations of the normalized average spectra for the longitudinal $(u)$, transversal $(v)$, and vertical $(w)$ wind velocity components at the Linhares tower. All these spectral curves have been averaged by the stability class. For all stability conditions, as the height increases, the spectral peak shifts to lower frequencies. However, this effect is more evident in the vertical velocity component, since large eddies in the 


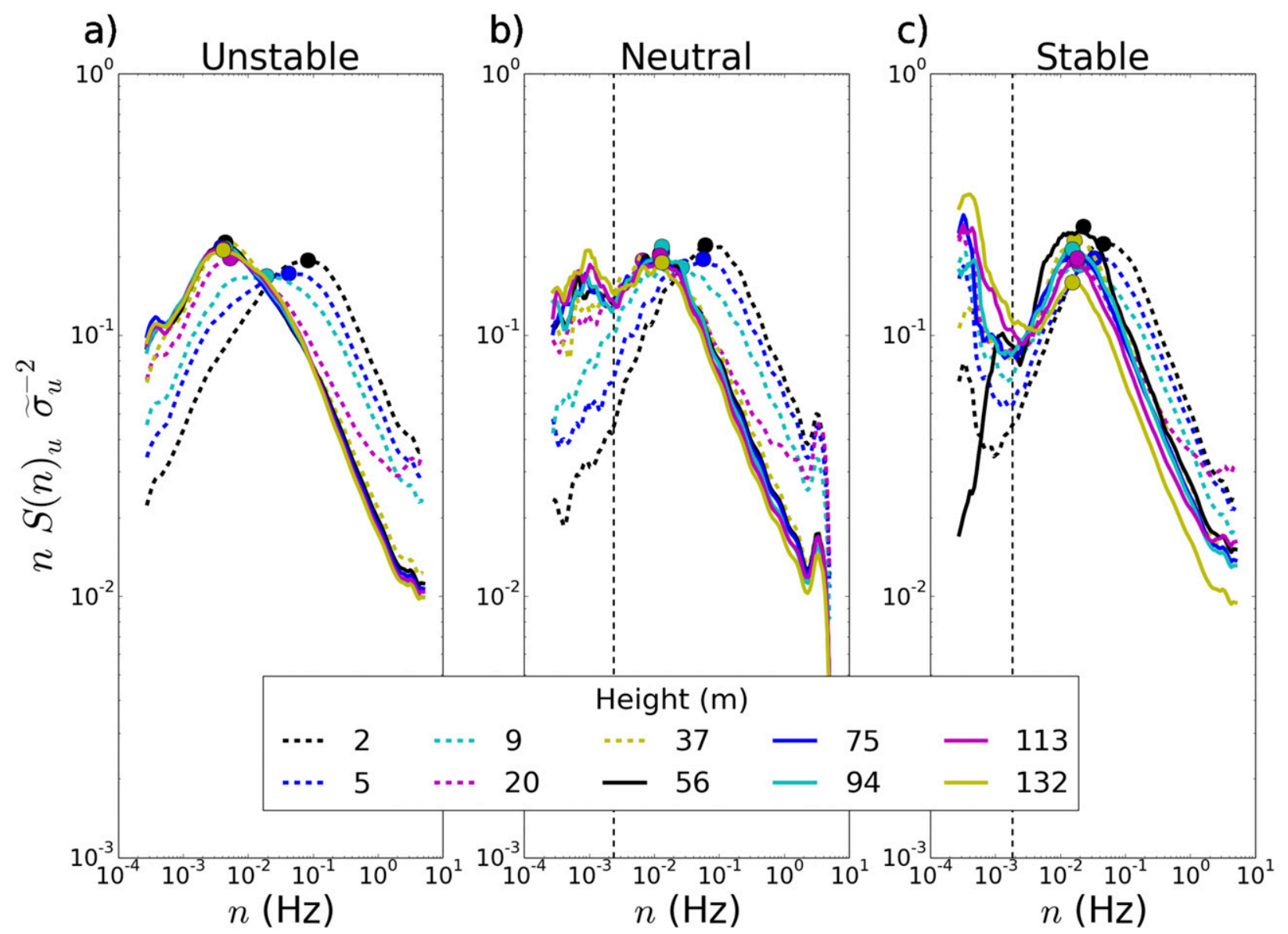

FIG. 1. Vertical variation of the normalized Hilbert-Huang marginal spectra (multiplied by frequency $n$ ) of the longitudinal component of the wind velocity averaged by the stability condition: (a) unstable, (b) neutral, and (c) stable. The spectra are normalized by $\tilde{\sigma}^{2}=\int_{n_{\min }}^{n_{\max }} S(n) d n$. Vertical lines represents the average gap scale.

vertical direction only exist in regions far from the surface. For unstable conditions, it can be seen that near the surface the $u$ - and $v$-component spectra in low frequencies already exhibit some effects of the large energetic convective eddies. This effect is more evident for the $v$ component, as found by Kaimal et al. (1972) and by Panofsky and Dutton (1983). At $9 \mathrm{~m}$, the spectral curve for the $v$ component is bimodal, with one peak associated with the convective forcing and the other associated with the mechanical forcing. As the height increases, the presence of the surface becomes less important, resulting in a decrease in the high-frequency spectral peak associated with the mechanical production, a phenomenon that has been previously observed by Kaimal et al. (1972) and Højstrup (1981). This fact shows that around $9 \mathrm{~m}$ there is an equilibrium between the convective turbulent energy and that mechanically generated by wind shear. For the $u$ component, the increase of the convective peak is more subtle, and the equilibrium between the mechanical and convective forcings is manifested by a plateau in the $9-\mathrm{m}$ observed spectra.

For both the $u$ and $v$ 1-h spectra (Figs. 1 and 2), in neutral and stable conditions, the presence of lowfrequency movements that do not constitute eddy motions is evident. These low-frequency motions can be associated with meso- and submesoscale motions (Mahrt 2008). To reduce the contribution of these largescale movements in the statistical turbulent parameters, the variance and the autocorrelation functions are evaluated over time periods that are on the order of the gap scale identified in the averaged spectra (Vickers and Mahrt 2003). For each horizontal component, an average gap scale was found for the neutral case and another for the stable case (Figs. 1b, 1c, 2b, and 2c). The standard deviations were then determined using time series with length equal to that gap scale, and averaged within each 1-h period. Although the gap scale is height dependent, for the purpose of calculating the variances, this difference can be neglected. Thus, the same time series length 


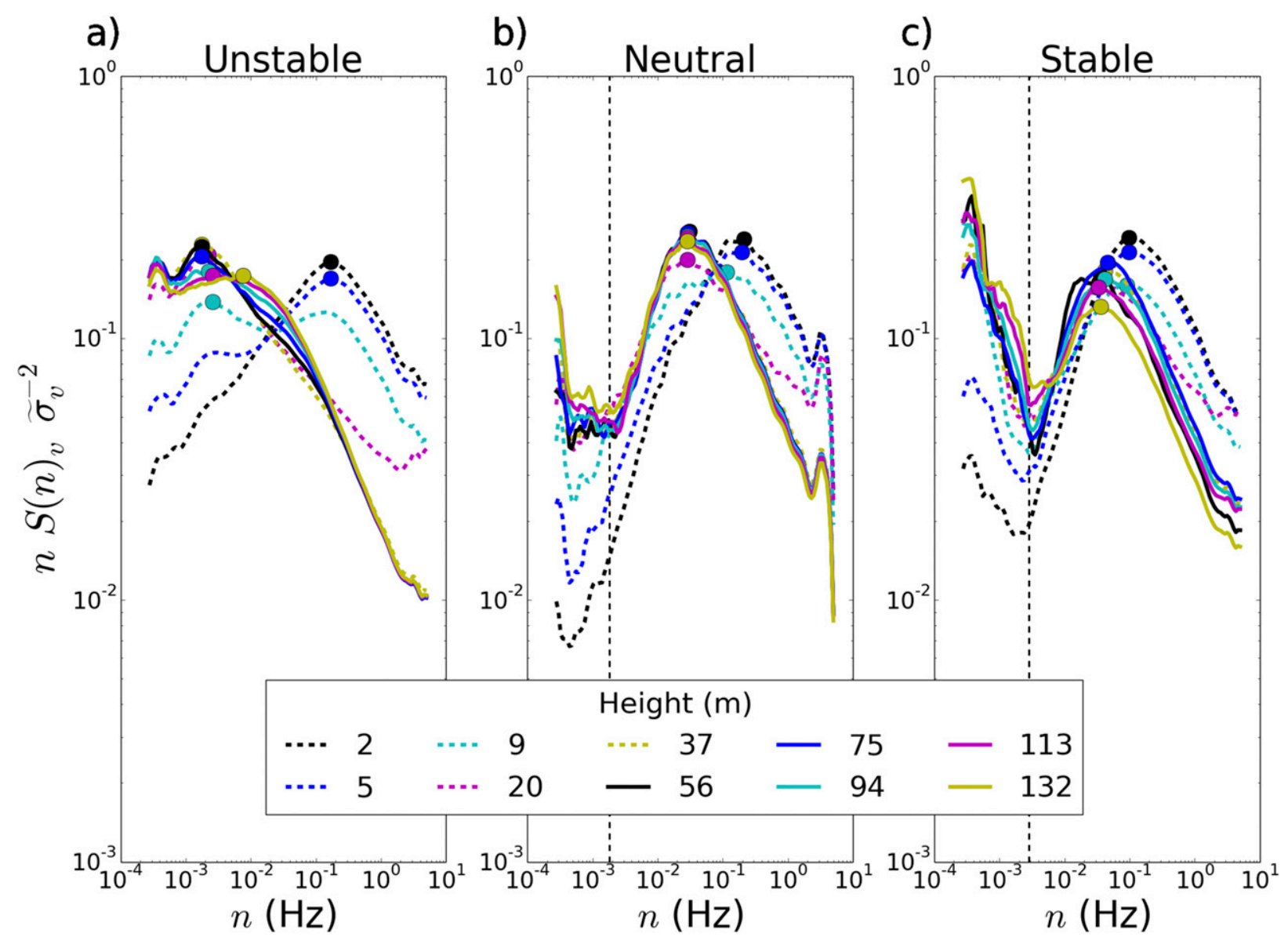

FIG. 2. As in Fig. 1, but for the transversal component of the wind velocity.

was used at all heights. When the gap is not evident, such for as the unstable case, and for the vertical component, the variances have been determined using the entire 1-h series. The gap scales found were $6 \mathrm{~min}$ for both the neutral and stable cases of the $u$ component, 4 min for the stable $v$ component, and $12 \mathrm{~min}$ for the neutral $v$ component. Neither the spectral peak (Fig. 4) nor the spectral peak frequency (Fig. 5) varies significantly within each stability class. There are some specific cases where these quantities deviate largely from the mean, especially in unstable conditions, but these outliers do not affect the averages. The decrease in the spectral peak frequency with height is evident in Fig. 5 for all wind components and stability conditions.

Figure 6 presents the vertical profile of the standard deviation $\left(\sigma_{i}\right)$ of the wind velocity components for each stability class. The continuous lines represent the averaged profiles of $\left(\sigma_{i}\right)$ evaluated using 1-h series while the dashed lines represent the values evaluated using shorter time series, based on the observed spectral gap scale. In the neutral cases, the short-window (sw) approach reduced the estimation of $\sigma_{i}$, in relation to the 1-h estimation, by $13 \%$ for the $u$ component and by $3 \%$ for the $v$ component. In the stable cases, the reductions were, respectively, $22 \%$ and $24 \%$ for the $u$ and $v$ components. The smallest difference occurs in the $v$ component under neutral conditions. This small difference occurs as a result of the reduced contribution of the low-frequency motions to the total energy compared to the other cases. To estimate $K_{\alpha}$ and $T_{L i}$, obtained from Eqs. (14) to (17), the short-window standard deviations have been used. To test the magnitude of the observed velocity standard deviations measured at 2-m height, Table 2 shows ratios between such standard deviations and the friction velocity at our tower compared to those found by other experimental results. For the neutral and stable conditions, the values observed at the Linhares tower are almost identical to those of Hanna (1982) and shown in Table 2. The observed values for the ratio $\sigma_{i} u_{*}^{-1}$ in the unstable case approach those found by Panofsky and Dutton (1983) and Arbage et al. (2008) in neutral situations. This agreement between the 


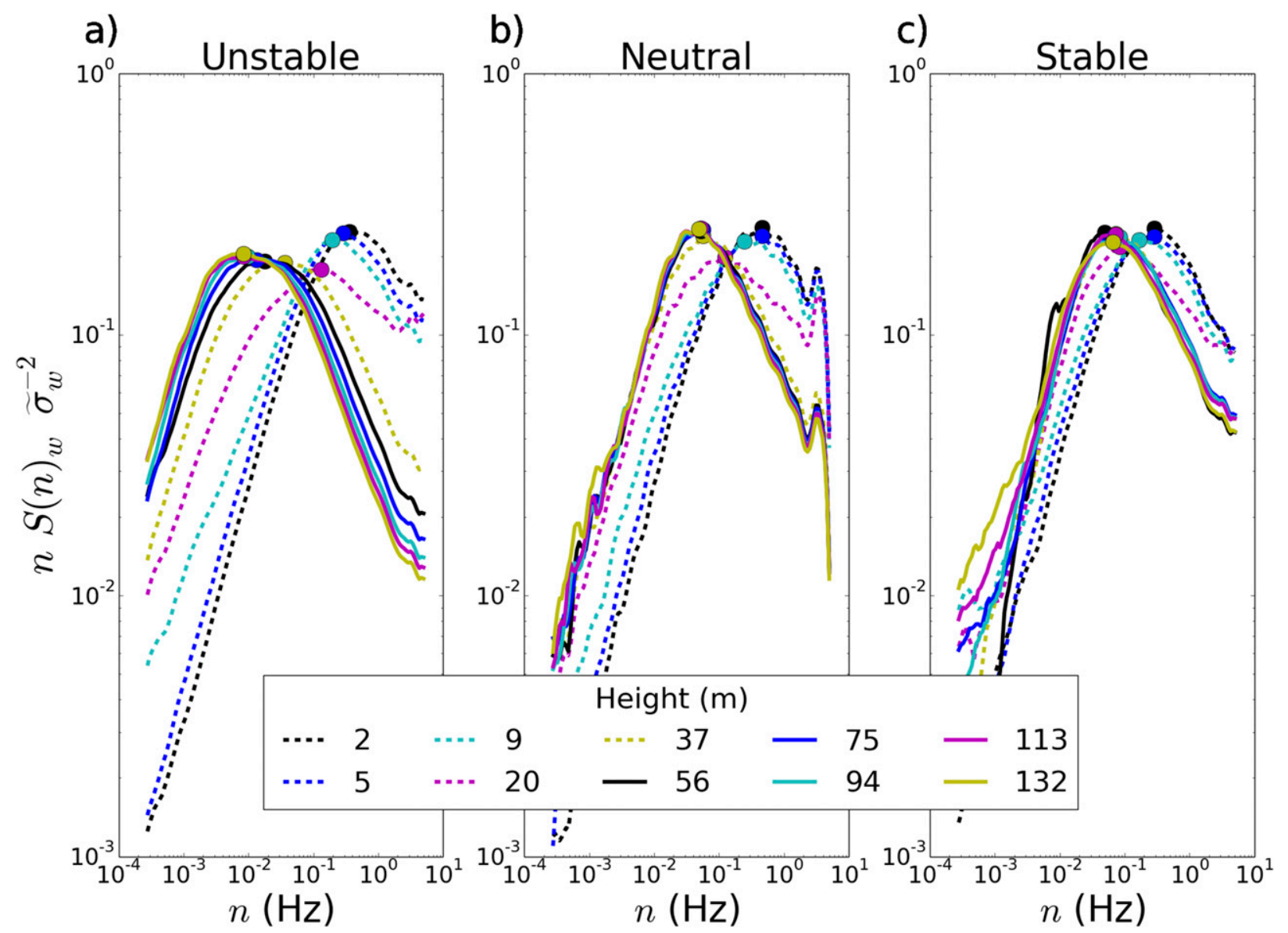

FIG. 3. As in Fig. 1, but for the vertical component of the wind velocity.

dimensionless velocity standard deviation in neutral and unstable cases confirms the dominant local effect played by the wind shear in the turbulence generation in the vertical regions of the PBL nearest to the surface.

These observed results for the turbulent velocity standard deviations and for the Hilbert-Huang marginal spectra, reproducing the characteristics observed in classical micrometeorological field experiments (Kaimal et al. 1972; Caughey and Palmer 1979), provide a reliable physical basis for calculating $K_{\alpha}$ and $T_{L i}$ from the Linhares tall micrometeorological tower turbulent data.

The vertical profiles of $T_{L i}$ for the different stability conditions are shown in Fig. 7, where the continuous lines represent the values obtained using the spectral formulation [Eqs. (16) and (17)] and the dashed lines represent those obtained directly from the Lagrangian autocorrelation function [Eqs. (18) and (2)]. For all stability conditions there is reasonably good agreement between the time scales obtained from the HilbertHuang spectra and those calculated directly from the autocorrelation functions. However, for the $u$ and $v$ unstable cases, in regions near the surface, there is some difference between the spectral and autocorrelation methods employed to calculate the time scales. Near the surface, the wind shear is the dominant process generating turbulence even in unstable conditions. According to Figs. 1a and 2a, these energetic mechanically generated eddies are associated with a high frequency $(0.1<n<1.0)$ spectral peak. For this reason, the unstable profiles of $T_{L i}$ near the ground are similar to those observed in the neutral and stable conditions, since $T_{L i}$ depends on $f_{m}$ [Eqs. (16) and (17)]. The neutral and stable time scales are independent of the determination method over the entire extension of the tower. The spectral method provides vertical time scales in good agreement with those obtained from the autocorrelation function method. For the horizontal components, the Degrazia et al. (2007) model indicates vertically constant values of $T_{L u}$ and $T_{L v}$ that are functions of the mixed layer depth $\left(z_{i}\right)$, convective velocity scale $\left(w_{*}\right)$, and dimensionless turbulence dissipation rate $(\psi)$. Acevedo et al. (2018) have shown that a thermal internal 
a) Unstable

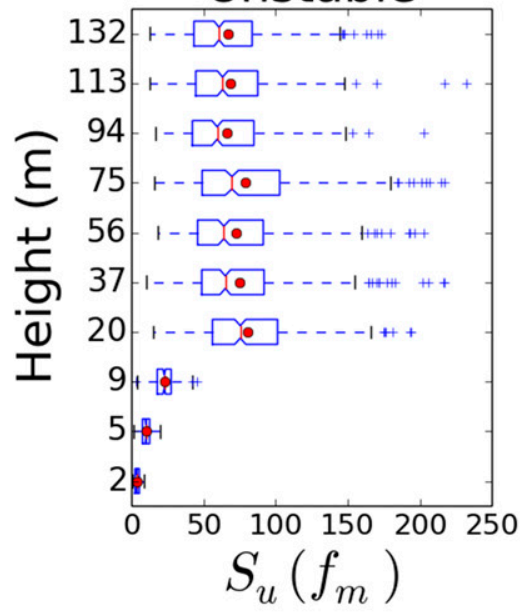

d)

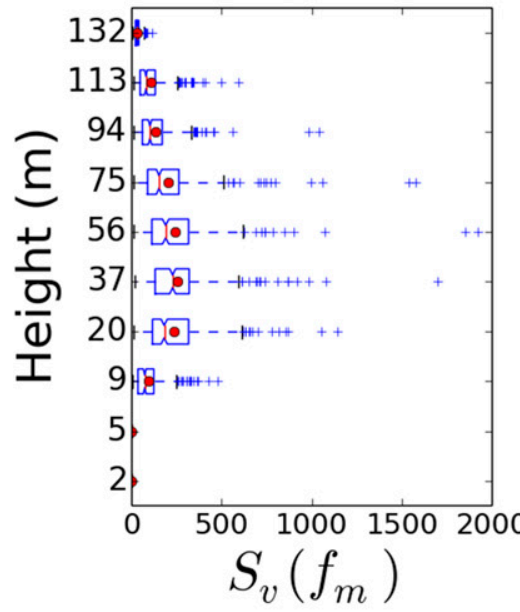

g)

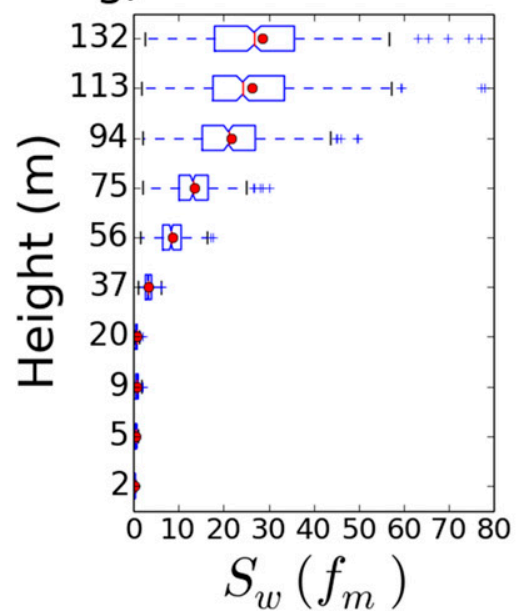

b) Neutral

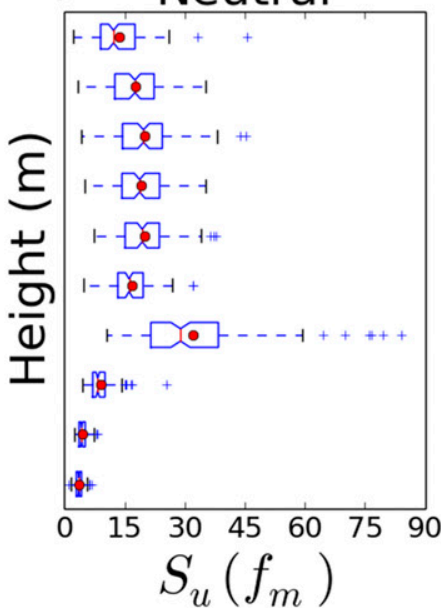

e)

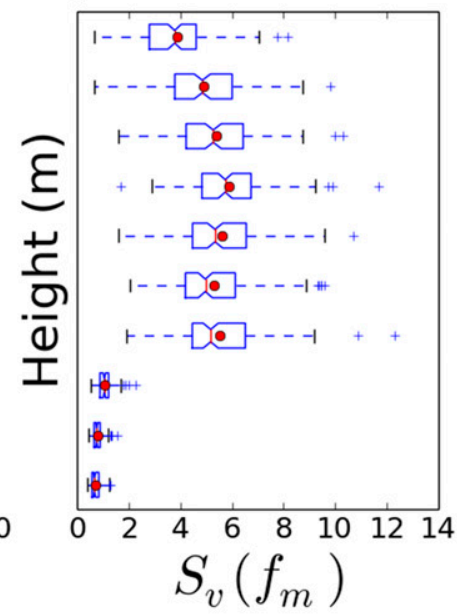

h)

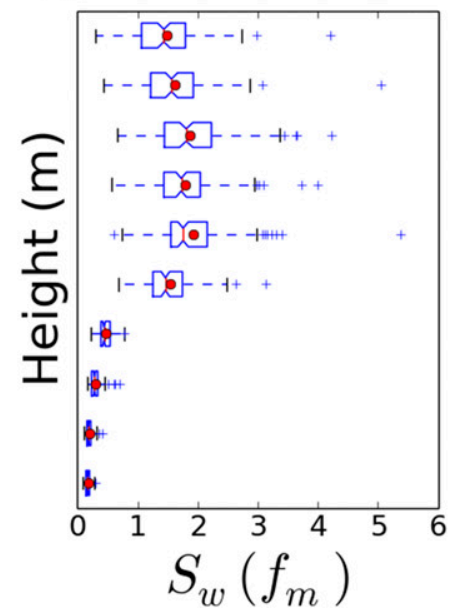

c) Stable

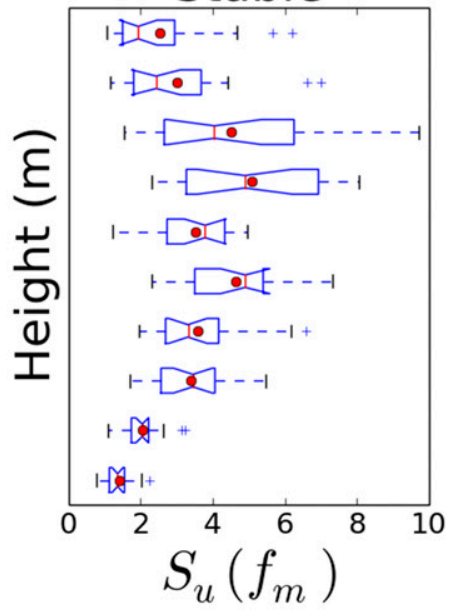

f)

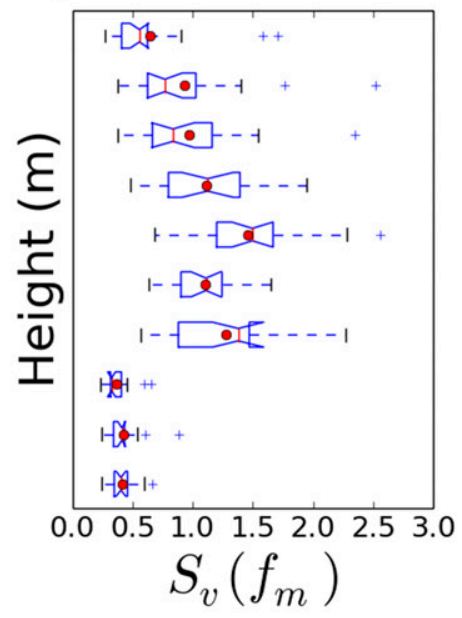

i)

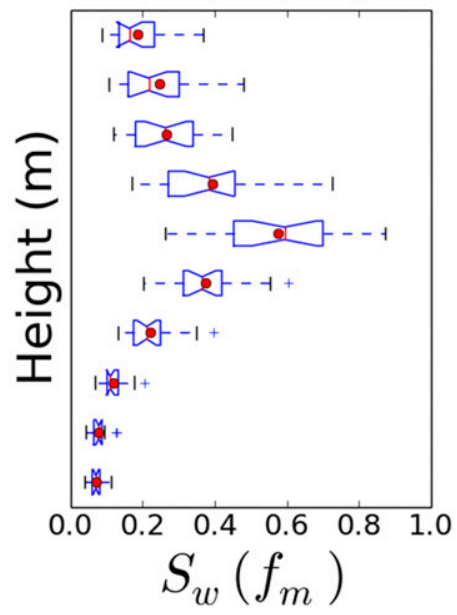

FIG. 4. Boxplots of the spectral energy at the frequency where the average spectrum has its maximum $\left(f_{m}\right)$ for different heights by stability condition: (a) unstable, (b) neutral, and (c) stable. The means are shown as red dots, while the medians are the short red vertical segments near the means. Outliers are shown by small plus signs. 


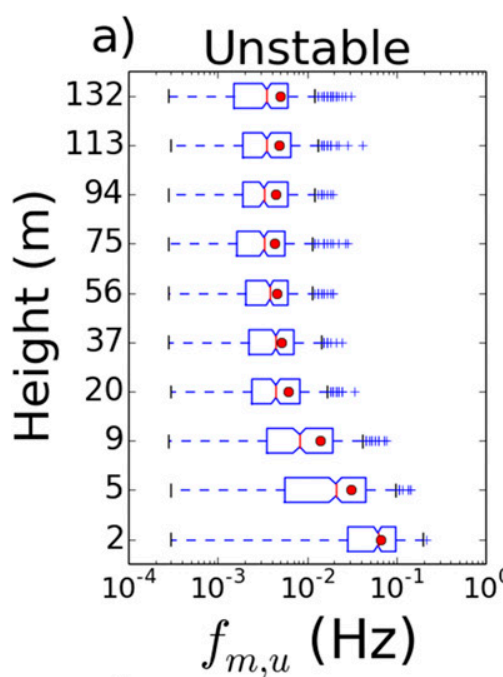

d)

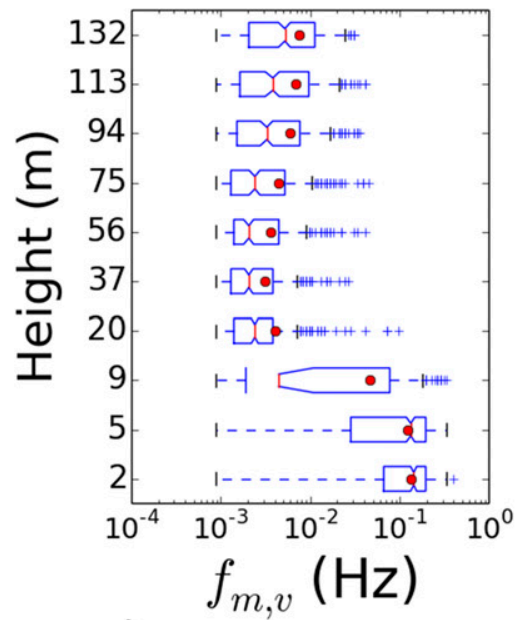

g)

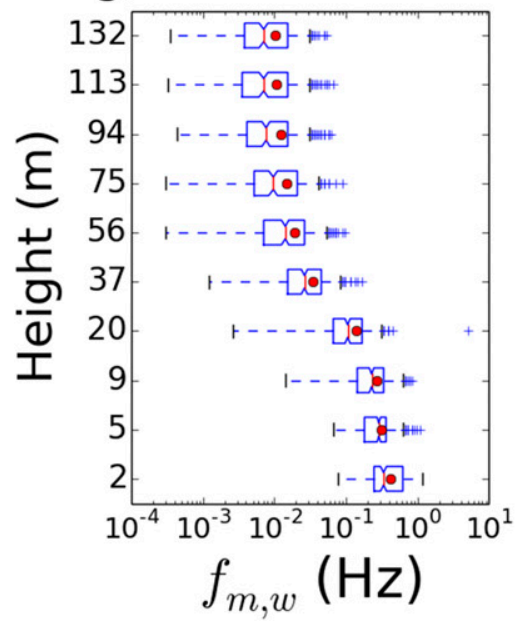

b) Neutral

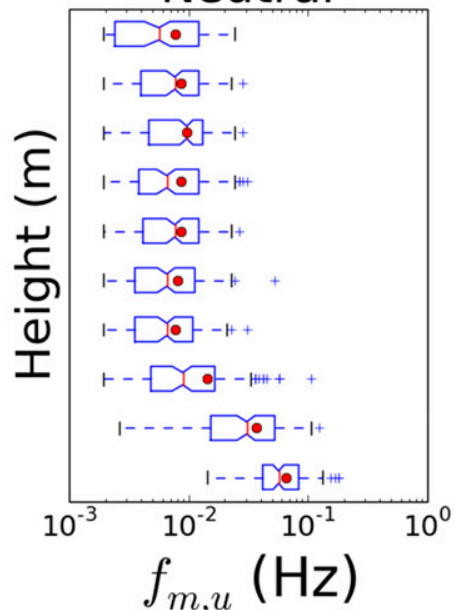

e)

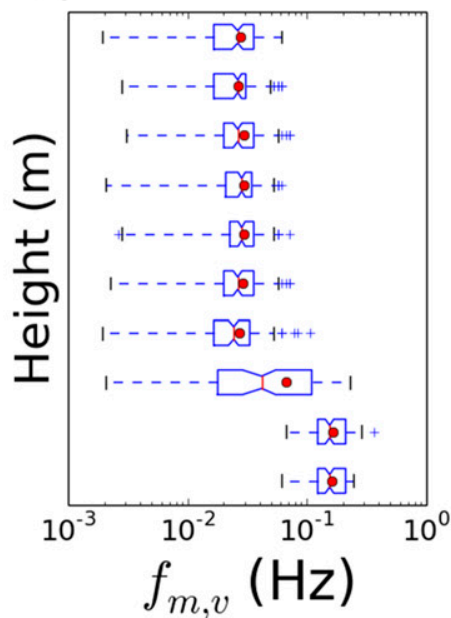

h)

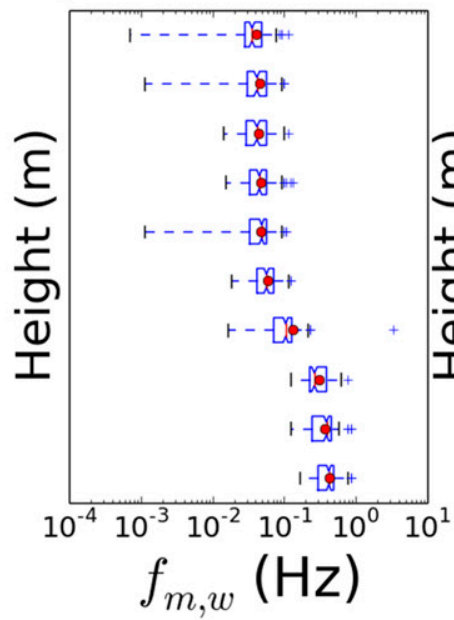

c) Stable

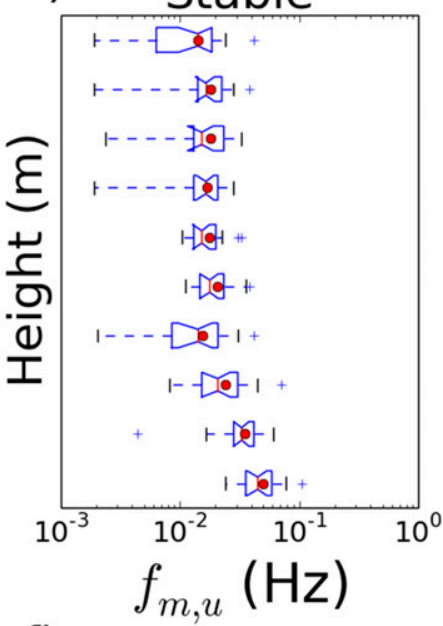

f)

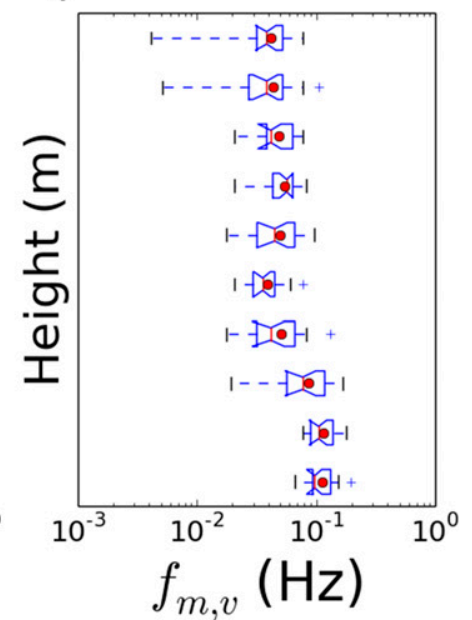

i)

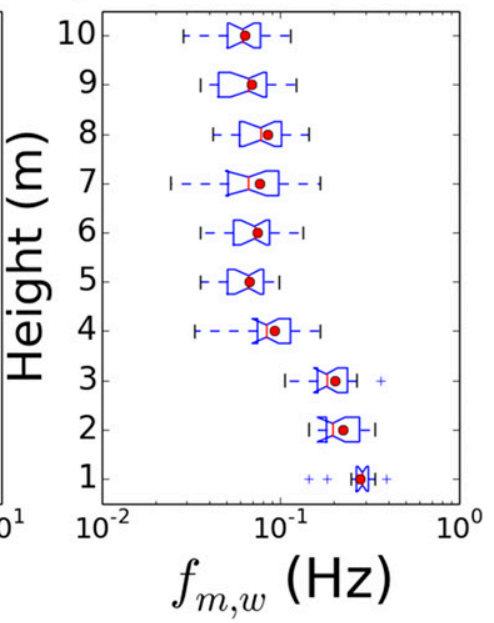

FIG. 5. As in Fig. 4, but for the spectral peak frequency $\left(f_{m}\right)$. 
a)

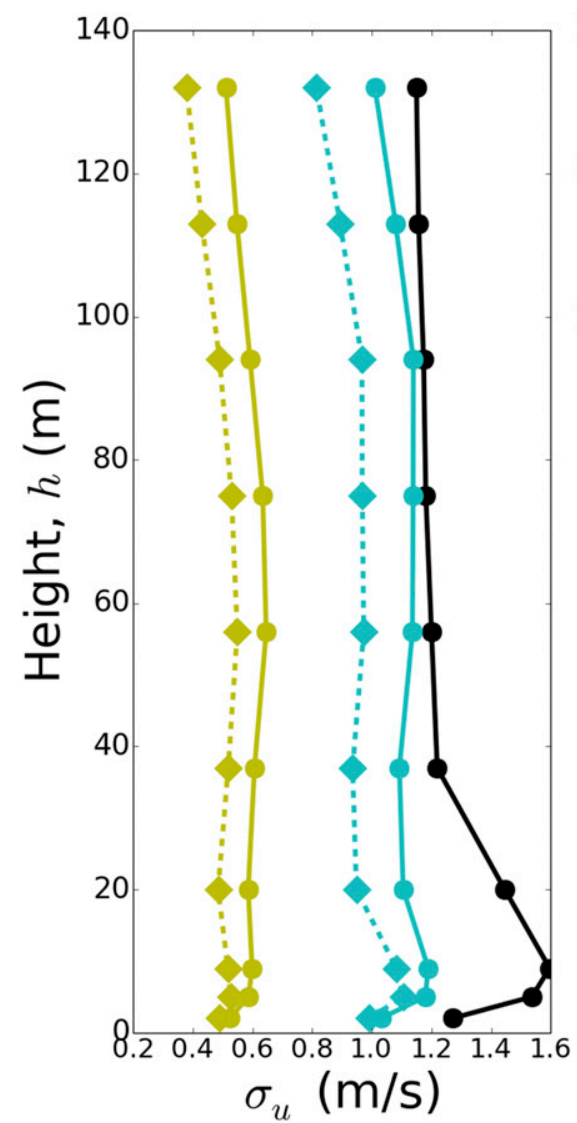

b)

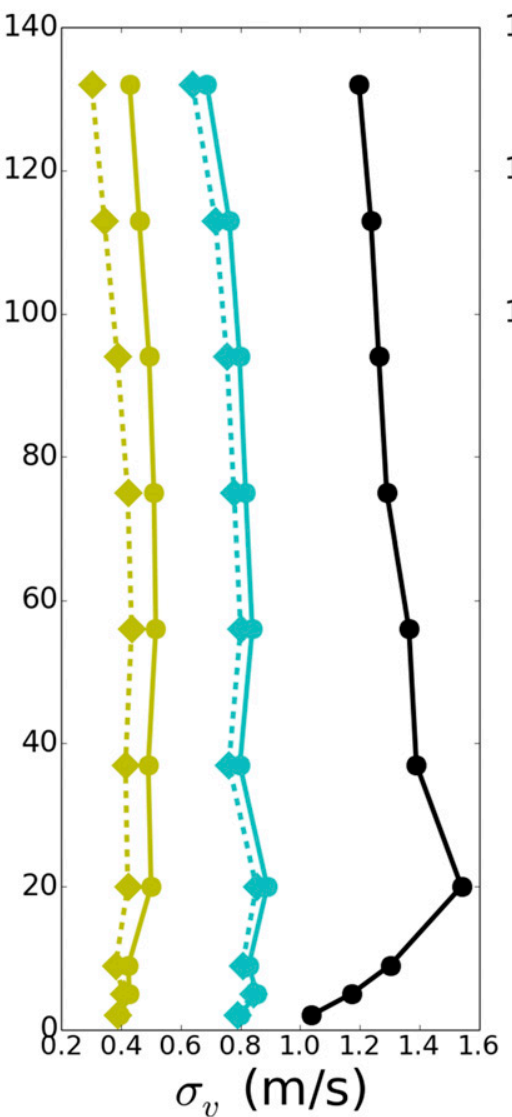

C)

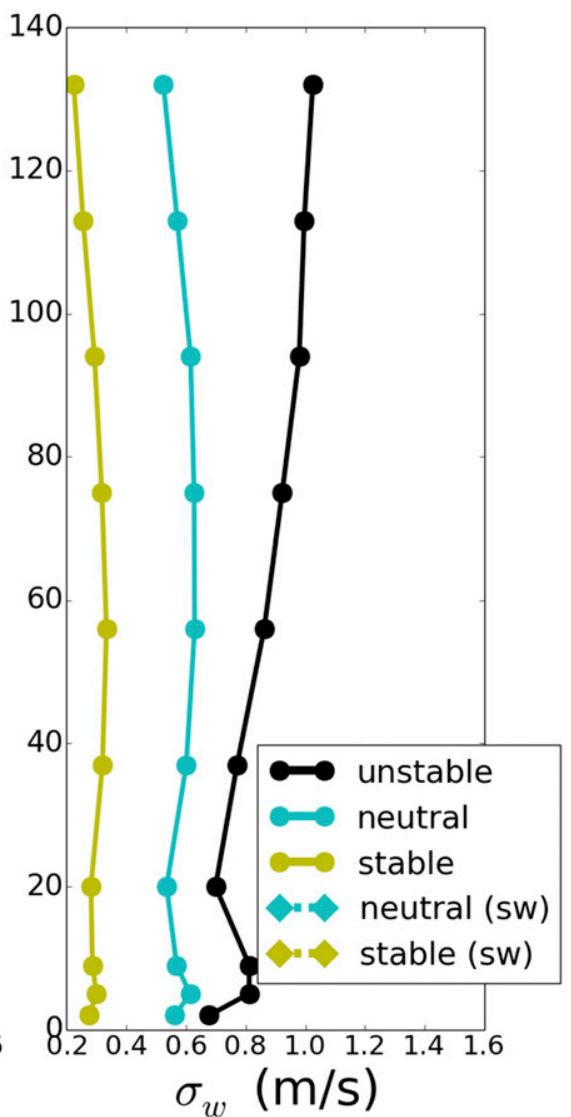

FIG. 6. Averaged vertical profile of the standard deviation $\left(\sigma_{i}\right)$ of the wind velocity components (a) $u$, (b) $v$, and (c) $w$ obtained for different stability classes. The continuous and dashed lines represent, respectively, the values determined using 1-h and short-window (sw) intervals.

boundary layer (TIBL) develops at the site during the afternoon. The vertical convergence of the sensible heat flux indicates that the depth of such a layer averages $367 \mathrm{~m}$ from 1200 to $1800 \mathrm{LST}$, in agreement with previous observations of TIBLs (Shao et al. 1991; Luhar et al. 1998). Acevedo et al. (2018) also showed that $w_{*}$ ranges between 1.1 and 1.5 during the same period. Using $z_{i}=367 \mathrm{~m}, w_{*}=1.35 \mathrm{~m} \mathrm{~s}^{-1}$, and the dissipation rates derived from the turbulence observations for the nondimensional molecular dissipation rate $\left(\psi^{1 / 3}=0.74\right)$, one estimates $T_{L u}=124 \mathrm{~s}$ and $T_{L v}=109 \mathrm{~s}$ (Table 3). The expression proposed by Weil et al. (2002) leads to slightly larger values (Table 3 ). Both expressions from Degrazia et al. (2007) and from Weil et al. (2002) underestimate the values obtained directly from the observations for the horizontal directions. On the other hand, for the vertical direction, the experimental profiles of convective $T_{L w}$ present a better level of agreement with the vertical profile derived by Degrazia et al. (2007) (Table 3).
The equivalence of the estimates of $T_{L i}$ obtained from spectral analysis with those obtained from the classical autocorrelation approach is relevant since various dispersion models use parameterizations based on energy spectra instead of autocorrelation functions (Ferrero et al. 1995; Ferrero and Anfossi 1998; Moreira et al. 2006a,b). This occurs because the spectra are better characterized/parameterized for the different conditions of stability than the autocorrelation functions. Magnitudes of the convective $T_{L v}$ calculated from the spectral method present larger values than those provided by the autocorrelation function method. This difference can be explained by the fact that, in this approach, $T_{L v}$ depends on the frequency of the spectral peak [Eq. (16)]. As shown in Fig. 2, the convective spectral peaks of the $v$ component are shifted toward lower frequencies. This effect increases $T_{L v}$ in comparison to $T_{L u}$. On the other hand, the values of $T_{L i}$ estimated from the autocorrelation functions depend on the global statistics of the turbulent components and, 
TABLE 2. Ratios between the standard deviation of the wind velocity components and the friction velocity observed at the Linhares tower and those presented by previous studies.

\begin{tabular}{|c|c|c|c|c|c|}
\hline & Height (m) & Condition & $\sigma_{u} u_{*}^{-1}$ & $\sigma_{v} u_{*}^{-1}$ & $\sigma_{w} u_{*}^{-1}$ \\
\hline Panofsky and Dutton (1983) & Various & Neutral & 2.39 & 1.92 & 1.25 \\
\hline Arbage et al. (2008) & 10 & Neutral & 2.67 & 1.95 & 1.39 \\
\hline Hanna (1982) & At the surface & Stable-neutral & 2.00 & 1.30 & 1.30 \\
\hline \multirow[t]{3}{*}{ Linhares } & 2 & Neutral & 2.06 & 1.64 & 1.17 \\
\hline & & Stable & 2.05 & 1.63 & 1.17 \\
\hline & & Unstable & 2.39 & 1.95 & 1.27 \\
\hline
\end{tabular}

therefore, $T_{L u}$ and $T_{L v}$ must present similar profiles, as occurs with the $\sigma_{u}$ and $\sigma_{v}$ profiles (Fig. 6). Given that the direction of the $u$ component is also where the turbulence shear production occurs, it may be speculated that turbulence dominates the flow in this direction, thus inhibiting lower-frequency motions, while in the crosswind direction such low-frequency perturbations persist in a more unaffected manner.
Generally, the magnitudes of $K_{x, y}$ for all stability cases initially increase with height in regions close to the surface and tend to average values that do not change significantly with height (Fig. 8). Furthermore, all $K_{\alpha}$ values are highly dependent on stability. Convective eddy diffusivities are larger than neutral ones and these in turn are larger than stable eddy diffusivities. Concerning the horizontal eddy diffusivities, the values of a)

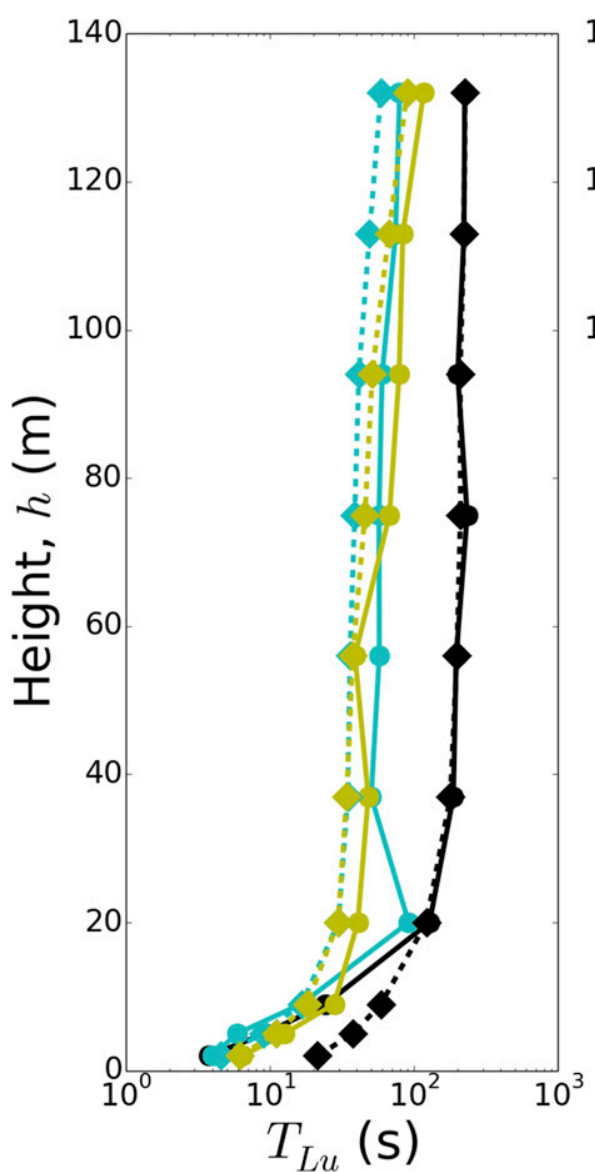

b)

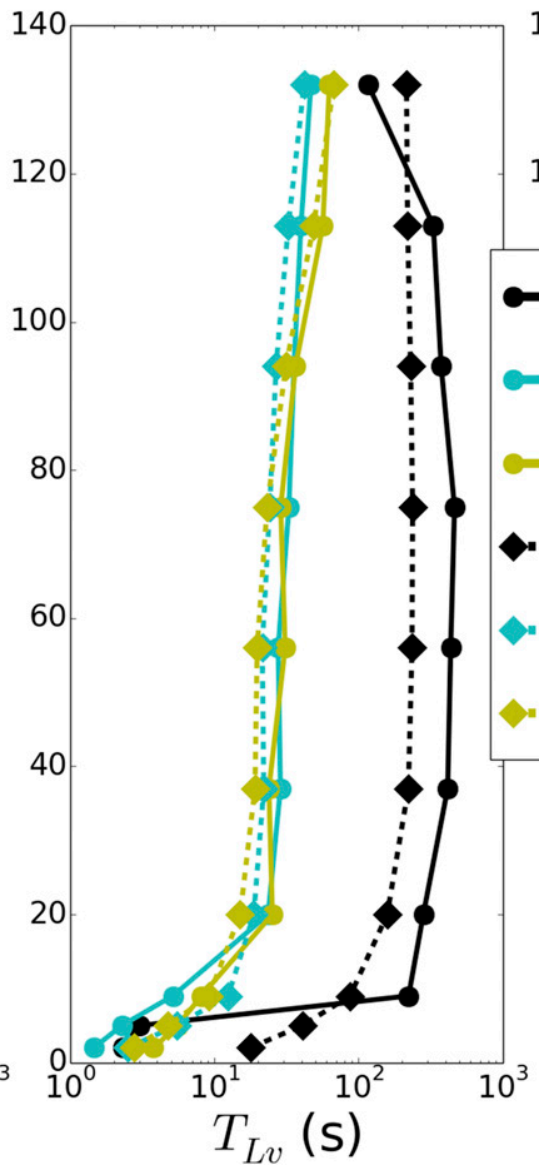

c)

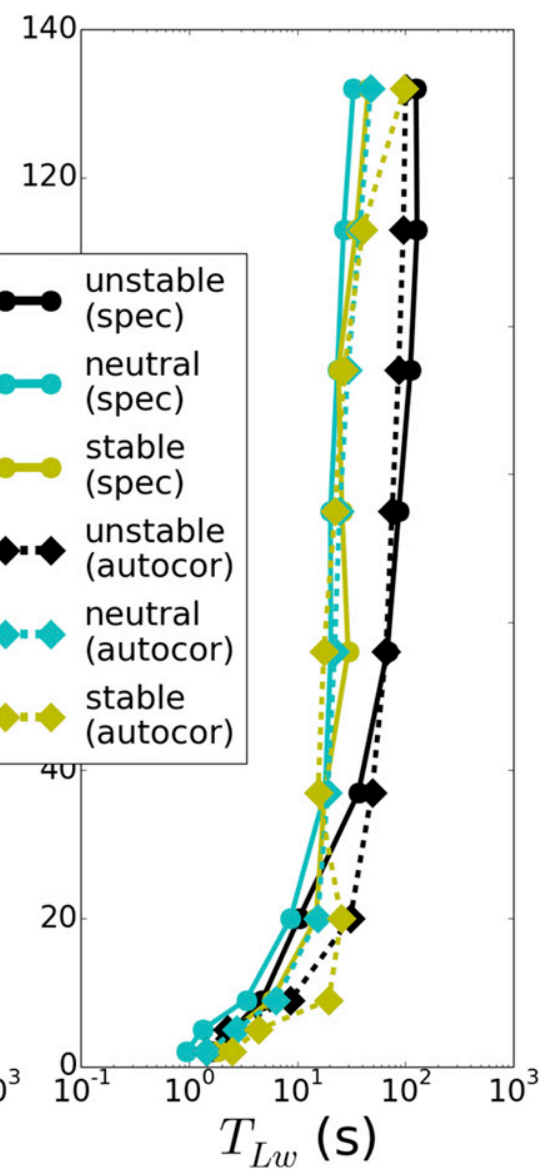

FIG. 7. As in Fig. 6, but for the local decorrelation time scales $\left(T_{L i}\right)$. The continuous and dashed lines represent, respectively, the values of $T_{L i}$ obtained using the spectral formulation [Eqs. (16) and (17)] and the autocorrelation approach [Eqs. (18) and (2)]. 
TABLE 3. Values of vertical and horizontal local decorrelation time scales $\left(T_{L u}, T_{L v}\right.$, and $\left.T_{L w}\right)$ estimated using both spectral [Eq. (16)] and autocorrelation [Eqs. (18) and (2)] approaches for the convective case. The experimental $T_{L u}$ and $T_{L v}$ time scales are averaged from the height of 20 to $140 \mathrm{~m}$. Values obtained from models derived by Degrazia et al. (2007) and Weil et al. (2002) are also provided.

\begin{tabular}{|c|c|c|c|c|}
\hline \multirow[b]{2}{*}{ Height } & $T_{L u}(\mathrm{~s})$ & $T_{L v}(\mathrm{~s})$ & \multirow{2}{*}{$\frac{T_{L w}(\mathrm{~s})}{20 \mathrm{~m}}$} & \multirow{2}{*}{$\frac{T_{L w}(\mathrm{~s})}{113 \mathrm{~m}}$} \\
\hline & \multicolumn{2}{|c|}{ Avg over $20 \mathrm{~m}$} & & \\
\hline Autocorrelation & 194 & 215 & 31 & 96 \\
\hline Spectral & 198 & 345 & 10 & 130 \\
\hline Degrazia et al. (2007) & 124 & 109 & 40 & 100 \\
\hline Weil et al. (2002) & 171 & 171 & - & - \\
\hline
\end{tabular}

$K_{x}$ and $K_{y}$ at heights higher than $20 \mathrm{~m}$ for the convective cases calculated from the autocorrelation method are on the order of $300 \mathrm{~m}^{2} \mathrm{~s}^{-1}$. The convective $K_{x}$ calculated from the spectral method presents the same magnitude. However, the lateral eddy diffusivity estimated from the spectral method exhibits magnitudes that are twice as large. The overestimation of $K_{y}$, likewise observed in the values of convective $T_{L v}$ obtained by the spectral method, results from the dependency of $K_{y}$ on the frequency of the spectral peak. In the lateral component, these are typically shifted to lower frequencies, increasing the magnitudes of $K_{y}$ with respect to $K_{x}$ (Kaimal et al. 1972). Following Wyngaard (1988), for the convective case an expression for the eddy diffusivities can be given by the relation $0.1 w_{*} z_{i}$ (Lamb and Durran 1978). As previously described, at the Linhares site these quantities average $w_{*}=1.35 \mathrm{~m} \mathrm{~s}^{-1}$ and $z_{i}=367 \mathrm{~m}$ in the afternoon, leading to an eddy diffusivity of $49.5 \mathrm{~m}^{2} \mathrm{~s}^{-1}$. This value is smaller than those obtained from the present analysis, of $280-375 \mathrm{~m}^{2} \mathrm{~s}^{-1}$ for the horizontal component and of $105-131 \mathrm{~m}^{2} \mathrm{~s}^{-1}$ for the vertical one (Table 4). Such discrepancies further indicate that expressions such as the one proposed by Lamb and Durran (1978) may be inappropriate for estimating the eddy diffusivities at internal boundary layers, such as that found at coastal regions.

In the neutral case, the lateral eddy diffusivities calculated from the autocorrelation and spectral methods are, respectively, 14 and $18 \mathrm{~m}^{2} \mathrm{~s}^{-1}$. On the other hand, the neutral longitudinal eddy diffusivities estimated from the autocorrelation and spectral method are, respectively, 35 and $50 \mathrm{~m}^{2} \mathrm{~s}^{-1}$. These values are in agreement with the $18 \mathrm{~m}^{2} \mathrm{~s}^{-1}$ result suggested by Garratt et al. (1992). Considering the characteristics of a moderately stable boundary layer (Table 1), the lateral eddy diffusivities estimated from the autocorrelation and spectral method are, respectively, 4.0 and $6.5 \mathrm{~m}^{2} \mathrm{~s}^{-1}$, while for the longitudinal eddy diffusivities the corresponding values are 11 and
$15 \mathrm{~m}^{2} \mathrm{~s}^{-1}$. Thus, the stable horizontal eddy diffusivities are on average 3 times smaller than those estimated for a neutral boundary layer.

The convective vertical eddy diffusivities calculated using the spectral and autocorrelation methods are shown in Fig. 8c. It can be seen that the vertical profile of $K_{z}$ for the unstable condition estimated from the spectral method increases with height, assuming values on the order of $3.1 \mathrm{~m}^{2} \mathrm{~s}^{-1}$ at $9 \mathrm{~m}, 74.0 \mathrm{~m}^{2} \mathrm{~s}^{-1}$ at $75 \mathrm{~m}$, and $131.9 \mathrm{~m}^{2} \mathrm{~s}^{-1}$ at $132 \mathrm{~m}$. For the same heights the magnitudes of unstable $K_{z}$ calculated from the autocorrelation method are, respectively, 5.7, 64.0, and $105.3 \mathrm{~m}^{2} \mathrm{~s}^{-1}$. Differently than for unstable $K_{z}$, the values of neutral and stable $K_{z}$ vary little with height. For the neutral case, the average value of $K_{z}$ for heights larger than $20 \mathrm{~m}$ is close to $9 \mathrm{~m}^{2} \mathrm{~s}^{-1}$ for both the spectral and autocorrelation methods. For the stable case, this mean value is near $3 \mathrm{~m}^{2} \mathrm{~s}^{-1}$.

At this point, to evaluate our vertical eddy diffusivities $\left(K_{z}\right)$ calculated by the spectral and autocorrelation methods, it is relevant to determine eddy diffusivities for momentum $\left(K_{m}\right)$, defined as

$$
K_{m}=\frac{-\overline{u^{\prime} w^{\prime}}}{\frac{d U}{d z}},
$$

in which the momentum flux $\left(\overline{u^{\prime} w^{\prime}}\right)$ and the mean wind speed gradient were provided by the micrometeorological tall-tower data. Following Wyngaard (2010), "the $K_{m}$ defined in Eq. (26) has not been documented extensively above the surface layer." Therefore, our data collected at vertical regions well above the surface layer provide a unique opportunity to obtain vertical profiles of $K_{m}$ in deep regions of the PBL. Figure 8c also exhibits the vertical profile of $K_{m}$ for distinct stability cases. For most levels, the gradients used in Eq. (26) have been determined from centered finite differences, considering observations at levels immediately above and below the level of consideration. For the first and last levels, they were evaluated from data at the level itself and, respectively, the level above or below it. The momentum flux used was that measured at the reference level. It can be seen that the vertical profile of $K_{m}$ in neutral conditions agrees fairly well with that of $K_{z}$, with an average value of $9 \mathrm{~m}^{2} \mathrm{~s}^{-1}$ above $20 \mathrm{~m}$. For stable conditions, there is reasonable agreement between the $K_{z}$ and $K_{m}$ profiles with $K_{m}$ assuming a mean value of $2 \mathrm{~m}^{2} \mathrm{~s}^{-1}$. For the convective case, $K_{z}$ and $K_{m}$ differ. This is a result of the flux gradient relationships not being valid in convective, well-mixed conditions when the gradients tend to zero. For this reason, a number of corrections have been proposed for the parameterization of 
a)

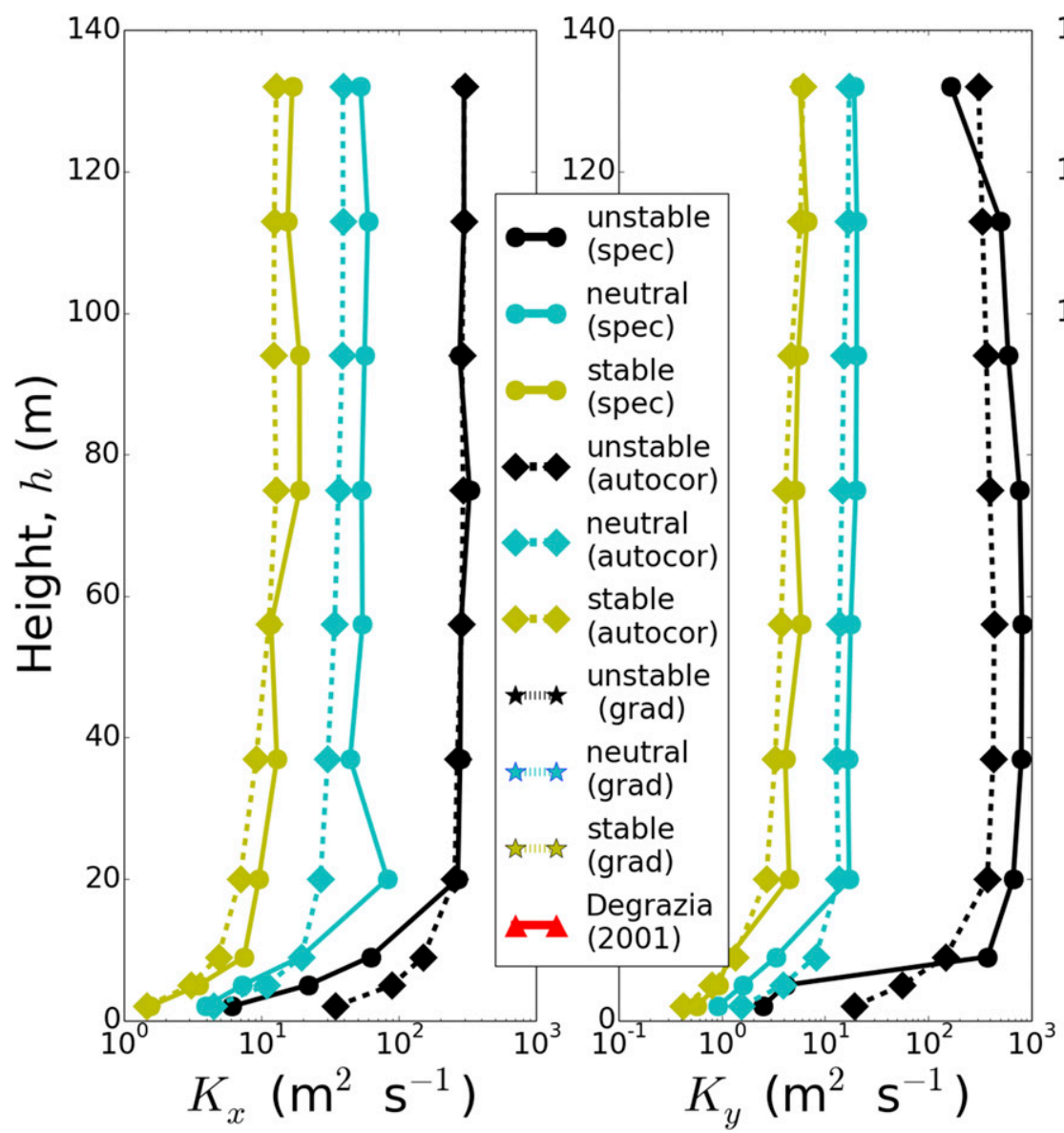

c)

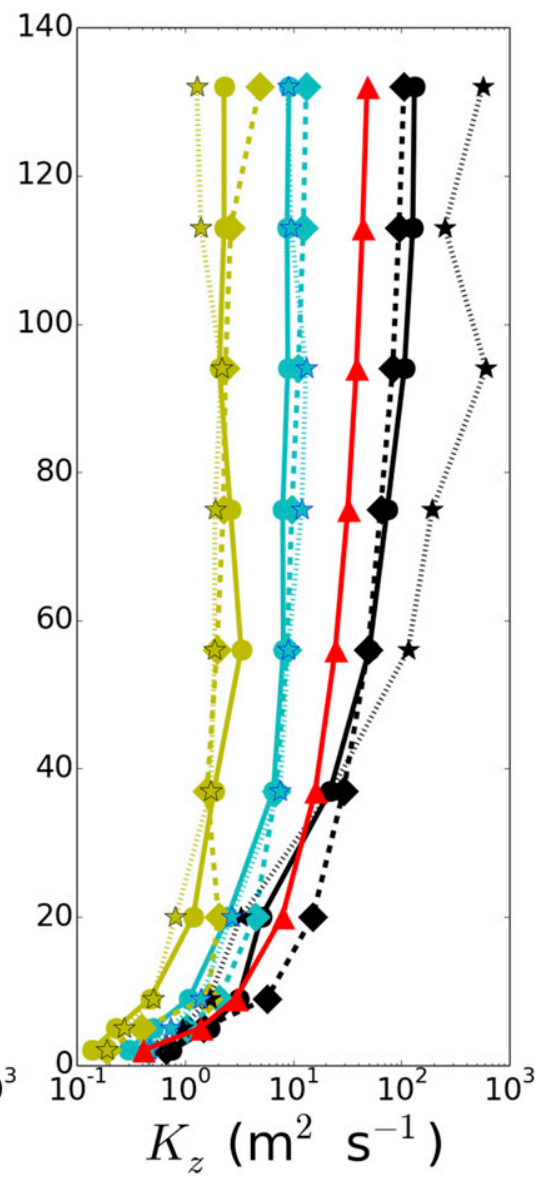

FIG. 8. As in Fig. 7, but for the eddy diffusivities $\left(K_{\alpha}\right)$ in the (a) $x$, (b) $y$, and (c) $z$ directions. The continuous and dashed lines represents, respectively, the values of $K_{\alpha}$ obtained using the spectral formulation [Eqs. (14) and (15)] and the autocorrelation approach [Eqs. (18) and (2)]. In (c), the dotted lines represent the eddy diffusivities for momentum evaluated directly from the flux-gradient relationship [Eq. (26)] and the red continuous line represents the vertical profile of the convective $K_{z}$ derived by Degrazia et al. (2001).

surface fluxes in convective conditions. In general, these corrections include contributions from nonlocal effects, such as convective large eddies to the local dispersion. For heat fluxes, for instance, this parameterization is referred to as the countergradient contribution (Holtslag and Moeng 1991). For momentum the same problem applies, but the solution is more complicated (Frech and Mahrt 1995). The $K_{m}$ value estimated from Eq. (26) corresponds only to the local contribution, as it depends only on local values of the wind shear and momentum flux. Frech and Mahrt (1995) stated that: "Purely local diffusion in the model simulation leads to a nonzero vertical gradient of the mean wind in order to produce nonzero stress everywhere within the boundary layer. In order to better compare with observations, the transport by the large boundary-layer eddies must be included in the mixing formulation for momentum as well as for heat and moisture." The $K_{z}$ formulation used [Eq. (14)], based on the spectral peak frequency, has a nonlocal character, as the largest convective eddies are responsible for the spectral peak. Therefore, based on the reasoning detailed above, it is expected that the $K_{z}$ formulation is better suited in convective conditions than the local $K_{m}$ values, at heights where the mixing activity destroys the local wind shear. As a test for our unstable $K_{z}$, we plot in Fig. 8c a mathematical formulation for the vertical profile of $K_{z}$. This formulation has been derived from the Taylor statistical diffusion theory and spectral empirical data (Degrazia et al. 2001) measured in Minnesota and Ashchurch, United Kingdom (Caughey and Palmer 1979). Using the characteristic values of $w_{*}=1.35 \mathrm{~m} \mathrm{~s}^{-1}, z_{i}=367 \mathrm{~m}$, and $\psi^{1 / 3}=0.74$, the magnitudes of $K_{z}$ obtained from this formulation at 9,75 , and $132 \mathrm{~m}$ are, respectively, 3, 32, and $48 \mathrm{~m}^{2} \mathrm{~s}^{-1}$, 
TABLE 4. Values of the stable (S), neutral $(\mathrm{N})$, and unstable $(\mathrm{U})$ eddy diffusivities $\left(K_{\alpha}\right)$ obtained from the Linhares dataset and those presented by previous studies.

\begin{tabular}{|c|c|c|c|c|c|c|c|c|c|c|c|}
\hline \multirow[b]{2}{*}{ Condition } & \multicolumn{3}{|c|}{$K_{x}\left(\mathrm{~m}^{2} \mathrm{~s}^{-1}\right)$} & \multicolumn{3}{|c|}{$K_{y}\left(\mathrm{~m}^{2} \mathrm{~s}^{-1}\right)$} & \multicolumn{5}{|c|}{$K_{z}\left(\mathrm{~m}^{2} \mathrm{~s}^{-1}\right)$} \\
\hline & $\mathrm{S}$ & $\mathrm{N}$ & $\mathrm{U}$ & $\mathrm{S}$ & $\mathrm{N}$ & $\mathrm{U}$ & $\mathrm{s}$ & $\mathrm{N}$ & & $\mathrm{U}$ & \\
\hline Height (m) & & & & Avg & $20 \mathrm{~m}$ & & & & 9 & 75 & 132 \\
\hline Spectral & 15 & 50 & 280 & 6.5 & 18 & 615 & 2.4 & 7.4 & 3.1 & 74 & 131.9 \\
\hline Autocorrelation & 11 & 35 & 280 & 4 & 14 & 375 & 3.6 & 9.5 & 5.7 & 64 & 105.3 \\
\hline Lamb and Durran (1978) & - & - & 49.5 & - & - & 49.5 & - & - & - & - & 49.5 \\
\hline Garratt et al. (1992) & 1.5 & 18 & 150 & 1.5 & 18 & 150 & - & - & - & - & 150 \\
\hline Degrazia et al. (2001) & - & - & - & - & - & - & - & - & 3.3 & 30.5 & 49.5 \\
\hline$K_{m}$ & - & - & - & - & - & - & 2 & 9 & - & - & - \\
\hline
\end{tabular}

which are in disagreement with the experimental convective $K_{z}$ evaluated from the Linhares tall-tower data (Fig. 8c). This difference indicates that the eddy diffusivities developed in coastal sites cannot be described by formulations derived from the turbulent parameters measured in continental regions.

In air pollution dispersion studies the $K_{z}$ eddy diffusivity is in some cases substituted by the $K_{m}$ eddy diffusivity in the diffusion-advection equation to simulate contaminant concentrations (Hanna 1982). This hypothesis is based in experimental evidences observed in regions near the surface. The present investigation, employing high-resolution turbulent observations measured in a tall micrometeorological tower, shows that this assumption is valid for vertical regions far from the surface both in neutral and stable conditions. This is an interesting result since the $K_{z}$ values, derived from the Taylor statistical diffusion model [Eq. (15)], are calculated as a product of the turbulent vertical velocity variance by the vertical decorrelation local time scales, while $K_{m}$ is based solely on the hypothesis of transport by the gradient [Eq. (26)]. Recently, Di Bernardino et al. (2017), investigating turbulent properties calculated from data obtained in a water channel, showed that $K_{z}$ evaluated from a Taylor model [Eq. (15)] agrees reasonably well with $K_{m}$ estimated from the first-order closure for the momentum flux [Eq. (26)], but no similar results had been provided for the lowest $140 \mathrm{~m}$ of the PBL in the field. Therefore, the present investigation helps to substantiate the employment of Eq. (26), which infers $K_{m}$ from measurements of wind speed gradients and friction velocity, in regions above the surface layer.

The values of $K_{\alpha}$ mentioned in the above discussion are summarized in Table 4.

\section{Conclusions}

Turbulent parameters such as eddy diffusivities $\left(K_{\alpha}\right)$, velocity variances $\left(\sigma_{i}\right)$, and Lagrangian local decorrelation time scales $\left(T_{L i}\right)$ for different stability conditions are fundamental input quantities in Eulerian and Lagrangian dispersion models. These dispersion models, incorporating well-adequated turbulent parameters, provide realistic simulated values for the atmospheric contaminant concentration. In this study, turbulent wind data measured at a tall micrometeorological tower situated at a coastal site along with velocity energy spectra, calculated from the Hilbert-Huang transform, have been used to obtain the Lagrangian local decorrelation time scales and eddy diffusivities from simple mathematical expressions [Eqs. (14)-(17)]. The main novelty is that the parameters are directly inferred from local observations at the tall tower. Furthermore, results from both spectral and autocorrelation analyses are compared.

The Lagrangian local decorrelation time scales, calculated from the energy spectra using Eqs. (16) and (17), present good agreement with those provided by the classical autocorrelation method. However, the occurrence of high-frequency energy peaks in surface vertical regions of the PBL associated with the shear turbulence generation in the $u$ and $v$ convective spectra is responsible for the difference between $T_{L u, v}$ obtained with the spectral method and results obtained using the autocorrelation method. Furthermore, for the entire vertical region covered by the tower, $T_{L v}$, calculated from the spectral method [Eq. (16)] is larger than that obtained from the autocorrelation method [Eqs. (18) and (2)]. This disagreement is caused by the fact that the unstable $v$ spectra present maximum values in lowerfrequency regions when compared with the $u$-component spectra. On the other hand, for $T_{L w}$ both methods provide similar magnitudes. Concerning applications in air pollution dispersion models, this equivalence between the two procedures used to determine $T_{L w}$ is particularly relevant since the turbulence in the vertical plane is the dominant physical mechanism of dispersion of atmospheric contaminants.

The eddy diffusivities calculated from the spectral and autocorrelation methods for horizontal and lateral directions, considering the distinct stability classes, 
increase with height in the lower levels, converging to a nearly constant value in the higher levels. These profiles of $K_{\alpha}$ are well grouped when described in terms of the different stability conditions with $K_{\alpha}^{s}<K_{\alpha}^{n}<K_{\alpha}^{c}$. The present analysis also shows that, in general, $K_{\alpha}^{n} \approx 3 K_{\alpha}^{s}$. The magnitudes of convective $K_{x, y}$ calculated from the autocorrelation method and the values of convective $K_{x}$ obtained from the spectral method are in good agreement with values on the order of $300 \mathrm{~m}^{2} \mathrm{~s}^{-1}$. On the other hand, $K_{z}^{c}$ magnitudes present outstanding growth with the distance from the surface. The unstable $K_{z}$ vertical profiles calculated from the autocorrelation and spectral methods do not agree well with the classical vertical profiles derived from the turbulent data measured in continental regions (far from the ocean). Therefore, a very important conclusion of the present study is that it is still necessary to develop specific models for describing the turbulent dispersion parameters at coastal sites.

Another important result generated by the measurements at different levels in the tall micrometeorological tower has been the finding that the vertical profiles for momentum eddy diffusivities $\left(K_{m}\right)$ are equivalent to those of $K_{z}$ estimated from the spectral and autocorrelation methods in neutral and stable conditions. Thus, turbulent parameters such as $\sigma_{i}, T_{L i}$, and $K_{\alpha}$, obtained in this work are found to be suitable for applications in atmospheric dispersion models.

Acknowledgments. This study has been developed within the context of a research and development project regulated by the Brazilian National Agency for Electric Energy and sponsored by the companies Linhares Geração S.A. and Termelétrica Viana S.A. The authors are gratefully indebted to these companies for all the support provided. The authors also thank the Coordenação de Aperfeiçoamento de Pessoal de Ensino Superior (CAPES, Brazil) for the financial support of this work.

\section{REFERENCES}

Acevedo, O. C., F. D. Costa, P. E. Oliveira, F. S. Puhales, G. A. Degrazia, and D. R. Roberti, 2014: The influence of submeso processes on stable boundary layer similarity relationships. J. Atmos. Sci., 71, 207-225, https://doi.org/10.1175/ JAS-D-13-0131.1.

— coastal site next to a thermal power plant from the surface to 140 m. Bull. Amer. Meteor. Soc., 99, 725-738, https://doi.org/ 10.1175/BAMS-D-17-0134.1.

Anfossi, D., and W. Physick, 2005: Lagrangian particle models. Air Quality Modeling: Theories, Methodologies, Computational Techniques and Available Databases and Software, P. Zannetti, Ed., EnvireComp Institute and Air \& Waste Management Association, 93-162.
Arbage, M. C. A., and Coauthors, 2008: Turbulent statistical characteristics associated to the north wind phenomenon in southern Brazil with application to turbulent diffusion. Physica $A$, 387, 4376-4386, https://doi.org/10.1016/j.physa.2008.02.068.

Batchelor, G., 1949: Diffusion in a field of homogeneous turbulence. I. Eulerian analysis. Aust. J. Chem., 2, 437-450, https:// doi.org/10.1071/CH9490437.

Blackadar, A. K., 1997: Turbulence and Diffusion in the Atmosphere: Lectures in Environmental Sciences. Springer, 185 pp.

Caughey, S., and S. Palmer, 1979: Some aspects of turbulence structure through the depth of the convective boundary layer. Quart. J. Roy. Meteor. Soc., 105, 811-827, https://doi.org/ 10.1002/qj.49710544606.

Champagne, F., C. Friehe, J. LaRue, and J. Wynagaard, 1977: Flux measurements, flux estimation techniques, and fine-scale turbulence measurements in the unstable surface layer over land. J. Atmos. Sci., 34, 515-530, https://doi.org/10.1175/ 1520-0469(1977)034<0515:FMFETA > 2.0.CO;2.

Degrazia, G., and D. Anfossi, 1998: Estimation of the Kolmogorov constant $C_{0}$ from classical statistical diffusion theory. Atmos. Environ., 32, 3611-3614, https://doi.org/10.1016/ S1352-2310(98)00038-7.

,-- J. Carvalho, C. Mangia, T. Tirabassi, and H. C. Velho, 2000a: Turbulence parameterisation for PBL dispersion models in all stability conditions. Atmos. Environ., 34, 35753583, https://doi.org/10.1016/S1352-2310(00)00116-3.

,,,--- H. C. Velho, E. Ferrero, C. Mangia, U. Rizza, and S. T. Castelli, 2000b: Turbulence parameterization for PBL dispersion models in all stability conditions. Air Pollution Modeling and Its Application XIII, S.-E. Gryning and E. Batchvarova, Eds., Springer, 745-746.

— D. M. Moreira, and M. T. Vilhena, 2001: Derivation of an eddy diffusivity depending on source distance for vertically inhomogeneous turbulence in a convective boundary layer. J. Appl. Meteor., 40, 1233-1240, https://doi.org/10.1175/15200450(2001)040<1233:DOAEDD > 2.0.CO;2.

_ J. C. Carvalho, D. M. Moreira, M. T. Vilhena, D. R. Roberti, and S. G. Magalhães, 2007: Derivation of a decorrelation timescale depending on source distance for inhomogeneous turbulence in a convective boundary layer. Physica A, 374, 5565, https://doi.org/10.1016/j.physa.2006.06.020.

Di Bernardino, A., P. Monti, G. Leuzzi, and G. Querzoli, 2017: Water-channel estimation of Eulerian and Lagrangian time scales of the turbulence in idealized two-dimensional urban canopies. Bound.-Layer Meteor., 165, 251-276, https://doi.org/ 10.1007/s10546-017-0278-6.

Ferrero, E., and D. Anfossi, 1998: Comparison of PDFs, closure schemes and turbulence parameterisations in Lagrangian stochastic models. Int. J. Environ. Pollut., 9, 384-410, https:// doi.org/10.1504/IJEP.1998.028253.

,,-- G. Brusasca, and G. Tinarelli, 1995: Lagrangian particle model LAMBDA: Evaluation against tracer data. Int. J. Environ. Pollut., 5, 360-374, https://doi.org/10.1504/IJEP.1995.028383.

Frech, M., and L. Mahrt, 1995: A two-scale mixing formulation for the atmospheric boundary layer. Bound.-Layer Meteor., 73, 91-104, https://doi.org/10.1007/BF00708931.

Garratt, J., and Coauthors, 1992: The Atmospheric Boundary Layer. Cambridge University Press, 444 pp.

Gifford, F. A., 1955: A simultaneous Lagrangian-Eulerian turbulence experiment. Mon. Wea. Rev., 83, 293-301, https://doi.org/ 10.1175/1520-0493(1955)083<0293:ASLTE > 2.0.CO;2.

Gryning, S.-E., P. Van Ulden, and R. E. Larsen, 1983: Dispersion from a continuous ground-level source investigated by a $\mathrm{K}$ 
model. Quart. J. Roy. Meteor. Soc., 109, 355-364, https://doi.org/ 10.1002/qj.49710946008.

Hanna, S. R., 1981: Lagrangian and Eulerian time-scale relations in the daytime boundary layer. J. Appl. Meteor., 20, 242-249, https:// doi.org/10.1175/1520-0450(1981)020<0242:LAETSR>2.0.CO;2.

_ - 1982: Applications in air pollution modeling. Atmospheric Turbulence and Air Pollution Modelling, F. T. M. Nieuwstadt and H. Van Dop, Eds., Reidel, 275-310.

Hinze, J., 1975: Turbulence. McGraw-Hill, 790 pp.

Højstrup, J., 1981: A simple model for the adjustment of velocity spectra in unstable conditions downstream of an abrupt change in roughness and heat flux. Bound.-Layer Meteor., 21, 341-356, https://doi.org/10.1007/BF00119278.

Holtslag, A., and C.-H. Moeng, 1991: Eddy diffusivity and countergradient transport in the convective atmospheric boundary layer. J. Atmos. Sci., 48, 1690-1698, https://doi.org/10.1175/ 1520-0469(1991)048<1690:EDACTI > 2.0.CO;2.

Huang, N. E., and Coauthors, 1998: The empirical mode decomposition and the Hilbert spectrum for nonlinear and nonstationary time series analysis. Proc. Roy Soc. London, 454A, 903-995, https://doi.org/10.1098/rspa.1998.0193.

_ Z Z. Shen, and S. R. Long, 1999: A new view of nonlinear water waves: The Hilbert spectrum. Annu. Rev. Fluid Mech., 31, 417-457, https://doi.org/10.1146/annurev.fluid.31.1.417.

Kaimal, J. C., and J. J. Finnigan, 1994: Atmospheric Boundary Layer Flows. Oxford University Press, 289 pp.

_ J. Wyngaard, Y. Izumi, and O. Coté, 1972: Spectral characteristics of surface-layer turbulence. Quart. J. Roy. Meteor. Soc., 98, 563-589, https://doi.org/10.1002/qj.49709841707.

,$- \ldots$, D. Haugen, O. Coté, Y. Izumi, S. Caughey, and C. Readings, 1976: Turbulence structure in the convective boundary layer. J. Atmos. Sci., 33, 2152-2169, https://doi.org/ 10.1175/1520-0469(1976)033<2152:TSITCB > 2.0.CO;2.

Lamb, R., and D. Durran, 1978: Eddy diffusivities derived from a numerical model of the convective planetary boundary layer. Nuovo Cimento C, 1, 1-17, https://doi.org/10.1007/BF02508043.

Leelössy, Á., F. Molnár, F. Izsák, Á. Havasi, I. Lagzi, and R. Mészáros, 2014: Dispersion modeling of air pollutants in the atmosphere: A review. Open Geosci., 6, 257-278, https:// doi.org/10.2478/s13533-012-0188-6.

Luhar, A., B. Sawford, J. Hacker, and K. Rayner, 1998: The Kwinana coastal fumigation study: II-Growth of the thermal internal boundary layer. Bound.-Layer Meteor., 89, 385-405, https://doi.org/10.1023/A:1001746303967.

Mahrt, L., 1998: Nocturnal boundary-layer regimes. Bound.-Layer Meteor., 88, 255-278, https://doi.org/10.1023/A:1001171313493.

_ 2008: The influence of transient flow distortion on turbulence in stable weak-wind conditions. Bound-Layer Meteor., 127, 1-16, https://doi.org/10.1007/s10546-007-9244-z.

Martins, L. G. N., and Coauthors, 2016: Employing the HilbertHuang transform to analyze observed natural complex signals: Calm wind meandering cases. Physica A, 462, 1189-1196, https://doi.org/10.1016/j.physa.2016.06.147.

Moreira, D. M., M. T. Vilhena, D. Buske, and T. Tirabassi, 2006a: The GILTT solution of the advection-diffusion equation for an inhomogeneous and nonstationary PBL. Atmos. Environ., 40, 3186-3194, https://doi.org/10.1016/j.atmosenv.2006.01.035.

,,-- T. Tirabassi, C. Costa, and B. Bodmann, 2006b: Simulation of pollutant dispersion in the atmosphere by the Laplace transform: The ADMM approach. Water Air Soil Pollut., 177, 411-439, https://doi.org/10.1007/s11270-006-9182-2.

Mortarini, L., M. Stefanello, G. Degrazia, D. Roberti, S. T. Castelli, and D. Anfossi, 2016: Characterization of wind meandering in low-wind-speed conditions. Bound.-Layer Meteor., 161, 165-182, https://doi.org/10.1007/s10546-016-0165-6.

Panofsky, H. A., and J. A. Dutton, 1983: Atmospheric Turbulence. John Wiley and Sons, $424 \mathrm{pp}$.

Pasquil, F., and F. Smith, 1983: Atmospheric Diffusion. 3rd ed. John Wiley and Sons, $437 \mathrm{pp}$.

Rilling, G., P. Flandrin, and P. Goncalves, 2003: On empirical mode decomposition and its algorithms. Proc. Workshop on Nonlinear Signal and Image Processing, Grado, Italy, IEEE-EURASIP, 8-11.

Rodean, H. C., 1996: Stochastic Lagrangian Models of Turbulent Diffusion. Meteor. Monogr., No. 48, Amer. Meteor. Soc., 84 pp.

Sawford, B., 1985: Lagrangian statistical simulation of concentration mean and fluctuation fields. J. Climate Appl. Meteor., 24, 1152-1166, https://doi.org/10.1175/1520-0450(1985)024<1152: $\mathrm{LSSOCM}>2.0 . \mathrm{CO} ; 2$

Shao, Y., J. M. Hacker, and P. Schwerdtfeger, 1991: The structure of turbulence in a coastal atmospheric boundary layer. Quart. J. Roy. Meteor. Soc., 117, 1299-1324, https://doi.org/10.1002/ qj. 49711750209.

Sharan, M., and M. Modani, 2006: A two-dimensional analytical model for the dispersion of air-pollutants in the atmosphere with a capping inversion. Atmos. Environ., 40, 3479-3489, https://doi.org/10.1016/j.atmosenv.2006.01.051.

Sorbjan, Z., 1989: Structure of the Atmospheric Boundary Layer. Prentice Hall, 317 pp.

Steeneveld, G., and A. Holtslag, 2009: Meteorological aspects of air quality. Air Quality in the 21st Century, G. C. Romano and A. G. Conti, Eds., Nova Science Publishers, 67-114.

Sun, J., L. Mahrt, R. M. Banta, and Y. L. Pichugina, 2012: Turbulence regimes and turbulence intermittency in the stable boundary layer during CASES-99. J. Atmos. Sci., 69, 338-351, https://doi.org/10.1175/JAS-D-11-082.1.

Thomson, D., 1987: Criteria for the selection of stochastic models of particle trajectories in turbulent flows. J. Fluid Mech., 180, 529-556, https://doi.org/10.1017/S0022112087001940.

Ulke, A. G., 2000: New turbulent parameterization for a dispersion model in the atmospheric boundary layer. Atmos. Environ., 34, 1029-1042, https://doi.org/10.1016/S1352-2310(99)00378-7.

Van de Wiel, B., A. Moene, and H. Jonker, 2012: The cessation of continuous turbulence as precursor of the very stable nocturnal boundary layer. J. Atmos. Sci., 69, 3097-3115, https:// doi.org/10.1175/JAS-D-12-064.1.

Vickers, D., and L. Mahrt, 2003: The cospectral gap and turbulent flux calculations. J. Atmos. Oceanic Technol., 20, 660-672, https:// doi.org/10.1175/1520-0426(2003)20<660:TCGATF $>2.0 . C O ; 2$.

Wandel, C., and O. Kofoed-Hansen, 1962: On the EulerianLagrangian transform in the statistical theory of turbulence. J. Geophys. Res., 67, 3089-3093, https://doi.org/10.1029/ JZ067i008p03089.

Weil, J. C., W. H. Snyder, R. E. Lawson, and M. S. Shipman, 2002: Experiments on buoyant plume dispersion in a laboratory convection tank. Bound.-Layer Meteor., 102, 367-414, https:// doi.org/10.1023/A:1013874816509.

Wyngaard, J. C., 1988: Structure of the PBL. Air Pollution Modeling, A. Venkatram and J. C. Wyngaard, Eds., Amer. Meteor. Soc., 9-57.

2010: Turbulence in the Atmosphere. Cambridge University Press, $406 \mathrm{pp}$.

Young, H.-W. V., K.-H. Hsu, V.-T. Pham, T.-T. Tran, and M.- T. Lo, 2017: A new approach to sparse decomposition of nonstationary signals with multiple scale structures using self-consistent nonlinear waves. Physica A, 481, 1-10, https:// doi.org/10.1016/j.physa.2017.04.009. 